\title{
On Hölder continuity of the solution of stochastic wave equations in dimension three
}

\author{
Yaozhong Hu • Jingyu Huang • David Nualart
}

Received: 28 August 2013 / Published online: 29 July 2014

(C) Springer Science+Business Media New York 2014

\begin{abstract}
In this paper, we study the stochastic wave equations in the three spatial dimensions driven by a Gaussian noise which is white in time and correlated in space. Our main concern is the sample path Hölder continuity of the solution both in time variable and in space variables. The conditions are given either in terms of the mean Hölder continuity of the covariance function or in terms of its spectral measure. Some examples of the covariance functions are proved to satisfy our conditions, which include the case of the work Dalang and Sanz-Solé (Hölder-Sobolev Regularity of the Solution to the Stochastic Wave Equation in Dimension Three. Memoirs of the American Mathematical Society Number 931 2009). In particular, we obtain the Hölder continuity results for the solution of the stochastic wave equations driven by (space inhomogeneous) fractional Brownian noises. For this particular noise, the optimality of the obtained Hölder exponents is also discussed.
\end{abstract}

Keywords Stochastic wave equation - Fractional Gaussian noises · Fourier transform · Spectral measure · Hölder continuity · Optimal Hölder exponent

\section{Introduction}

We shall study the following stochastic wave equation in spatial dimension $d=3$ :

$$
\left\{\begin{array}{l}
\left(\frac{\partial^{2}}{\partial t^{2}}-\Delta\right) u(t, x)=\sigma(t, x, u(t, x)) \dot{W}(t, x)+b(t, x, u(t, x)) \\
u(0, x)=v_{0}(x), \quad \frac{\partial u}{\partial t}(0, x)=\bar{v}_{0}(x)
\end{array}\right.
$$

Y. Hu $\cdot$ J. Huang $(\varangle) \cdot$ D. Nualart

Department of Mathematics, University of Kansas, Lawrence, KS 66045, USA

e-mail: huangjy@ku.edu 
where $t \in(0, T]$ for some fixed $T>0, x \in \mathbb{R}^{3}$ and $\Delta=\frac{\partial^{2}}{\partial x_{1}^{2}}+\frac{\partial^{2}}{\partial x_{2}^{2}}+\frac{\partial^{2}}{\partial x_{3}^{2}}$ denotes the Laplacian on $\mathbb{R}^{3}$. The coefficients $\sigma$ and $b$ satisfy some regularity conditions which will be specified later. The Gaussian noise process $\dot{W}$ is assumed to be white in time and with a homogeneous correlation in space. This can be informally written as

$$
\mathbb{E}[\dot{W}(t, x) \dot{W}(s, y)]=\delta(t-s) f(x-y)
$$

for a non-negative, non-negative definite and locally integrable function $f$, where $\delta$ is the Dirac delta function. We will explain in Sect. 2 how this expression can be made formal.

It is known (see, for instance, [4, Theorem 4.3]) that if $\sigma$ and $b$ are Lipschitz functions with linear growth and $f$ satisfies $\int_{|x| \leq 1} f(x) /|x| d x<\infty$, then there is a unique mild solution to Eq. (1.1). Our purpose is to establish the sample path Hölder continuity both in time variable and in space variables of the solution to this equation. When $f$ is given by a Riesz kernel $|x|^{-\beta}, \beta \in(0,2)$, the Hölder continuity of the solution has been obtained by Dalang and Sanz-Solé in their monograph [5]. Their approach is based on the fractional Sobolev imbedding theorem and the Fourier transformation technique.

In this paper, we shall consider more general Gaussian noises, and we introduce a new approach that avoids the Fourier transform. The main idea is to impose conditions on the covariance $f$ itself. To be more precise, let $D_{w} f=f(\cdot+w)$ be the shift operator. We shall show that if $\left\|D_{w} f-f\right\|_{L^{1}(\rho)} \leq C|w|^{\gamma}$ and $\left\|D_{w} f+D_{-w} f-2 f\right\|_{L^{1}(\rho)} \leq$ $C|w|^{\gamma^{\prime}}$ for some $\gamma \in(0,1]$ and $\gamma^{\prime} \in(0,2]$, where $\rho$ is the measure on $\mathbb{R}^{3}$ defined to be $\rho(d z)=\mathbf{1}_{\{|z| \leq 2 T\}} \frac{1}{|z|} d z$, then the solution to (1.1) is locally Hölder continuous of order $\kappa<\min \left(\gamma, \frac{\gamma^{\prime}}{2}\right)$ in the space variable (assuming zero initial conditions) (see Theorem 3.1).

The Hölder continuity in the time variable is more involved. Following the methodology used by Dalang and Sanz-Solé in [5], we transform the time increments into space increments, and we impose suitable assumptions on the modulus of continuity of a shift operator which are formulated integrals over $[0, T] \times\left(S^{2}\right)^{2}$, equipped with the measure $d s \sigma(d \xi) \sigma(d \eta)$, where $\sigma$ is the uniform measure on the unit sphere $S^{2}$ (see Theorem 4.1).

We also obtain a theorem on the Hölder continuity in the space variable using the Fourier transform technique. More precisely, we establish the Hölder continuity of order $\kappa<\gamma$, provided the spectral measure $\mu$ satisfies the integrability condition $\int_{\mathbb{R}^{3}} \frac{\mu(d \zeta)}{1+|\zeta|^{2-2 \gamma}}<\infty$ and the Fourier transform of $|\zeta|^{2 \gamma} \mu(d \zeta)$ is non-negative. The nonnegativity condition on this measure leads to a simple proof of the Hölder continuity in the space variable which avoids the control of the norms of the increments $D_{w} f-f$ and $D_{w} f+D_{-w} f-2 f$ (or their respective Fourier transforms). As an application, this method provides a direct proof of the Hölder continuity in the space variable, in the case of the Riesz kernel. However, at this moment we are not able to use this approach to handle the Hölder continuity in the time variable.

To illustrate the scope of our results we provide some examples of covariance functions $f$ which satisfy our conditions. We consider first the Riesz and Bessel 
kernels. Then we focus our attention to fractional noises with covariance function of the form

$$
f(x)=\left|x_{1}\right|^{2 H_{1}-2}\left|x_{2}\right|^{2 H_{2}-2}\left|x_{3}\right|^{2 H_{3}-2},
$$

where $H_{1}, H_{2}, H_{3} \in(1 / 2,1)$ and $\bar{\kappa}:=\sum_{i=1}^{3} H_{i}-2$. We show (see Theorem 6.1) that, under suitable assumptions on the initial conditions, if $\kappa_{i} \in\left(0, \min \left(H_{i}-1 / 2, \bar{\kappa}\right)\right)$ and $\kappa_{0}=\min \left(\kappa_{1}, \kappa_{2}, \kappa_{3}\right)$, then for any bounded rectangle $I \subset \mathbb{R}^{3}$, there is a finite random variable $K$, depending on the $\kappa_{i}$ 's, such that for all $s, t \in[0, T]$ and for all $x, y \in I$

$$
|u(t, x)-u(s, y)| \leq K_{I}\left(\left|x_{1}-y_{1}\right|^{\kappa_{1}}+\left|x_{2}-y_{2}\right|^{\kappa_{2}}+\left|x_{3}-y_{3}\right|^{\kappa_{3}}+|s-t|^{\kappa_{0}}\right) .
$$

To see if the Hölder exponents $\kappa_{i}$ 's are optimal or not, we investigate a simple linear stochastic wave equation with additive noise. That means, we consider the Eq. (1.1) with $v_{0}=\bar{v}_{0}=0, b=0$ and $\sigma=1$. In this situation, we prove (see Theorem 6.2 and a Kolomogorov lemma) that for any bounded rectangle $I \subset \mathbb{R}^{3}$ and for any $\kappa \in(0, \bar{\kappa})$, there is a random variable $K_{\kappa, I}$ such that for alll $t, s \in[0, T]$ and for all $x, y \in I$

$$
|u(t, x)-u(s, y)| \leq K_{\kappa, I}\left(\left|x_{1}-y_{1}\right|^{\kappa}+\left|x_{2}-y_{2}\right|^{\kappa}+\left|x_{3}-y_{3}\right|^{\kappa}+|s-t|^{\kappa}\right) .
$$

On the other hand, we obtain in Theorem 6.2 a lower bound on the variance of the increments of the process $u$ which shows that the exponent $\bar{\kappa}$ is optimal. Notice that in the nonlinear case (see Theorem 6.1), we need the extra conditions $\kappa_{i}<H_{i}-1 / 2$ for $i=1,2,3$. Also, this extra condition is not necessary if $H_{i}+H_{j} \leq 3 / 2$ for any $i \neq j$ (for instance, if $H_{1}=H_{2}=H_{3}=H \leq 3 / 4$ ), and in this case $\kappa_{i}$ coincides with the optimal constant $\bar{\kappa}$. It would be interesting to know if the additional conditions $\kappa_{i}<H_{i}-1 / 2$ are due to the nonlinearity or due to the limitation of our technique.

This paper is organized as follows. Section 2 contains some preliminary material about the noise process in Eq. (1.1). We state our basic assumptions on the covariance function $f$ and prove a general Burkholder inequality. We also give the definition of the mild solution and state the existence and uniqueness theorem of the solution to Eq. (1.1). Section 3 contains two main results on the Hölder continuity in the space variables. One is based on the structure of the covariance function $f$ itself and the other one uses the Fourier transform of $f$. In Sect. 4 we prove a criterion for the Hölder continuity in the time variable. Section 5 presents some examples of covariance functions $f$ which satisfy the conditions given in our main theorems. In the first example, $f$ is the convolution of a Schwartz function with a Riesz kernel. In the second example, $f$ is the Riesz kernel, which is the case studied in [5]. In the third example, $f$ is the Bessel kernel. Section 6 deals with the case when the noise process is the formal derivative of a fractional Brownian field. The optimality of the Hölder exponents is discussed in this section. Section 6 contains some lemmas which are used in the paper. 


\section{Preliminaries}

Consider a non-negative and non-negative definite function $f$ which is a tempered distribution on $\mathbb{R}^{3}$ (so $f$ is locally integrable). We know that in this case $f$ is the Fourier transform of a non-negative tempered measure $\mu$ on $\mathbb{R}^{3}$ (called the spectral measure of $f$ ). That is, for all $\varphi$ belonging to the space $\mathcal{S}\left(\mathbb{R}^{3}\right)$ of rapidly decreasing $C^{\infty}$ functions

$$
\int_{\mathbb{R}^{3}} f(x) \varphi(x) d x=\int_{\mathbb{R}^{3}} \mathcal{F} \varphi(\xi) \mu(d \xi),
$$

and there is an integer $m \geq 1$ such that

$$
\int_{\mathbb{R}^{3}}\left(1+|\xi|^{2}\right)^{-m} \mu(d \xi)<\infty,
$$

where we have denoted by $\mathcal{F} \varphi$ the Fourier transform of $\varphi \in \mathcal{S}\left(\mathbb{R}^{3}\right)$, given by

$$
\mathcal{F} \varphi(\xi)=\int_{\mathbb{R}^{3}} \varphi(x) e^{-i \xi \cdot x} d x .
$$

Let $G(t)$ be the fundamental solution of the 3-dimensional wave equation $\frac{\partial^{2} u}{\partial t^{2}}=\Delta u$. That is

$$
G(t)=\frac{1}{4 \pi t} \sigma_{t}
$$

for any $t>0$, where $\sigma_{t}$ denotes the uniform surface measure (with total mass $4 \pi t^{2}$ ) on the sphere of radius $t>0$. Sometimes it is more convenient for us to use the Fourier transform of $G$ given by

$$
\mathcal{F} G(t)(\xi)=\frac{\sin (t|\xi|)}{|\xi|}, \quad t>0
$$

Our basic assumption on $f$ is

$$
\int_{|x| \leq 1} \frac{f(x)}{|x|} d x<\infty
$$

It turns out (see Lemma 6.4 and Eq. (6.21) below) that this is equivalent to

$$
\int_{\mathbb{R}^{3}} \frac{\mu(d \xi)}{1+|\xi|^{2}}<\infty .
$$

Notice that since we are in $\mathbb{R}^{3}$, condition (2.5) is satisfied if there is a $\kappa<2$ such that in a neighborhood of $0, f(x) \leq C|x|^{-\kappa}$. 
The following identities will play an important role,

$$
\begin{aligned}
\frac{1}{8 \pi} \int_{s-t \leq|x| \leq s+t} \frac{f(x)}{|x|} d x & =\int_{\mathbb{R}^{3}}[G(s) * G(t)](x) f(x) d x \\
& =\int_{\mathbb{R}^{3}} \mu(d \xi)(\mathcal{F} G(s))(\xi)(\mathcal{F} G(t))(\xi)
\end{aligned}
$$

for $0<t \leq s$. We refer to Lemmas 6.4 and 6.5 for proofs of these two identities.

Fix a time interval $[0, T]$. Let $C_{0}^{\infty}\left([0, T] \times \mathbb{R}^{3}\right)$ be the space of infinitely differentiable functions with compact support on $[0, T] \times \mathbb{R}^{3}$. Consider a zero mean Gaussian family of random variables $W=\left\{W(\varphi), \varphi \in C_{0}^{\infty}\left([0, T] \times \mathbb{R}^{3}\right)\right\}$, defined in a complete probability space $(\Omega, \mathcal{F}, P)$, with covariance

$$
\mathbb{E}(W(\varphi) W(\psi))=\int_{0}^{T} \int_{\mathbb{R}^{3}} \int_{\mathbb{R}^{3}} \varphi(t, x) f(x-y) \psi(t, y) d x d y d t
$$

Walsh's classical theory of stochastic integration developed in [8] cannot be applied directly to the mild formulation of Eq. (1.1) since $G$ is not a function, but a measure. We shall use the stochastic integral defined in Sect. 2.3 of [4]. We briefly summarize the construction and properties of this integral.

Let $U$ be the completion of $C_{0}^{\infty}\left(\mathbb{R}^{3}\right)$ endowed with the inner product

$$
\langle\varphi, \psi\rangle_{U}=\int_{\mathbb{R}^{3}} d x \int_{\mathbb{R}^{3}} d y \varphi(x) f(x-y) \psi(y)=\int_{\mathbb{R}^{3}} \mathcal{F}(\varphi)(\xi) \overline{\mathcal{F}(\psi)(\xi)} \mu(d \xi),
$$

$\varphi, \psi \in C_{0}^{\infty}\left(\mathbb{R}^{3}\right)$. Set $U_{T}=L^{2}([0, T] ; U)$.

The Gaussian family $W$ can be extended to the space $U_{T}$. We will also denote by $W(g)$ the Gaussian random variable associated with an element $g \in U_{T}$. Set $W_{t}(h)=W\left(\mathbf{1}_{[0, t]} h\right)$ for any $t \in[0, T]$ and $h \in U$. Then $W=\left\{W_{t}, t \in[0, T]\right\}$ is a cylindrical Wiener process in the Hilbert space $U$. That is, for any $h \in U$, $\left\{W_{t}(h), t \in[0, T]\right\}$ is a Brownian motion with variance $t\|h\|_{U}^{2}$, and

$$
\mathbb{E}\left(W_{t}(h) W_{s}(g)\right)=(s \wedge t)\langle h, g\rangle_{U}
$$

Let $\mathcal{F}_{t}$ be the $\sigma$-field generated by the random variables $\left\{W_{s}(h), h \in U, 0 \leq s \leq t\right\}$ and the $P$-null sets. We define the predictable $\sigma$-field as the $\sigma$-field in $\Omega \times[0, T]$ generated by the sets $\left\{A \times(s, t], 0 \leq s<t \leq T, A \in \mathcal{F}_{s}\right\}$. Then we can define the stochastic integral of a $U$-valued square-integrable predictable process $g \in L^{2}(\Omega \times[0, T] ; U)$ 
with respect to the cylindrical Wiener process $W$, denoted by

$$
g \cdot W=\int_{0}^{T} \int_{\mathbb{R}^{3}} g(t, x) W(d t, d x)
$$

and we have the isometry property

$$
\mathbb{E}|g \cdot W|^{2}=\mathbb{E} \int_{0}^{T}\|g(t)\|_{U}^{2} d t
$$

The following lemma provides a sufficient condition for a measure of the form $\varphi(x) G(t, d x)$ to be in the space $U$.

Lemma 2.1 Consider a Borel measurable function $\varphi: \mathbb{R}^{3} \rightarrow \mathbb{R}$, such that for some $t>0$

$$
\int_{\mathbb{R}^{3}} \int_{\mathbb{R}^{3}}|\varphi(x) \varphi(y)| G(t, d x) G(t, d y) f(x-y)<\infty .
$$

Then, $\varphi G(t)$ belongs to $U$ and

$$
\|\varphi G(t)\|_{U}^{2}=\int_{\mathbb{R}^{3}} \int_{\mathbb{R}^{3}} \varphi(x) \varphi(y) G(t, d x) G(t, d y) f(x-y) .
$$

Furthermore, when $\varphi$ is bounded,

$$
\|\varphi G(t)\|_{U}^{2}=\int_{\mathbb{R}^{3}}|\mathcal{F}(\varphi G(t))(\xi)|^{2} \mu(d \xi)
$$

Proof Suppose first that $\varphi$ is bounded. Then by Lemma 6.5, the equality (2.13) holds and $\int_{\mathbb{R}^{3}}|\mathcal{F}(\varphi G(t))(\xi)|^{2} \mu(d \xi)<\infty$. Let $\psi$ be a nonnegative $C^{\infty}$ function on $\mathbb{R}^{3}$ supported in the unit ball such that $\int_{\mathbb{R}^{3}} \psi(x) d x=1$. Define $\psi_{n}(x)=n^{3} \psi(n x)$, so

$$
\left(\psi_{n} *(\varphi G(t))\right)(x):=\int_{\mathbb{R}^{3}} \psi_{n}(x-y) \varphi(y) G(t, d y)
$$

is in $C_{0}^{\infty}\left(\mathbb{R}^{3}\right)$, and we have 


$$
\begin{aligned}
& \int_{\mathbb{R}^{3}}\left|\mathcal{F}\left(\psi_{n} *(\varphi G(t))-\varphi G(t)\right)\right|^{2} \mu(d \xi) \\
& \quad=\int_{\mathbb{R}^{3}}\left|\left(\mathcal{F} \psi_{n}\right)(\xi)-1\right|^{2}|\mathcal{F}(\varphi G(t))(\xi)|^{2} \mu(d \xi) \rightarrow 0
\end{aligned}
$$

as $n \rightarrow \infty$, by the dominated convergence theorem. This implies that $\varphi G(t)$ is in $U$, and (2.12) holds.

In the general case, we consider the sequence of functions $\varphi_{k}(x)=\varphi(x) \mathbf{1}_{\{|\varphi| \leq k\}}$. Then $\varphi_{k}(x) G(t, d x)$ belongs to $U$, and

$$
\begin{aligned}
& \left\|\varphi_{k}(x) G(t, d x)-\varphi(x) G(t, d x)\right\|_{U}^{2} \\
& \leq \int_{\mathbb{R}^{3}} \int_{\mathbb{R}^{3}}\left|\varphi_{k}(x)-\varphi(x) \| \varphi_{k}(y)-\varphi(y)\right| G(t, d x) G(t, d y) f(x-y),
\end{aligned}
$$

which clearly goes to 0 as $k$ goes to infinity, by the dominated convergence theorem.

For any $x \in \mathbb{R}^{3}$ we denote by $G(t, x-d y)$ the shifted measure $A \mapsto G(t, x-A)$. Clearly Lemma 2.1 holds if we replace the kernel $G(t, d y)$ by the shifted kernel $G(t, x-d y)$. Applying Lemma 2.1, we immediately get the following Burkholder inequality.

Lemma 2.2 Let $Z=\left\{Z(t, x),(t, x) \in[0, T] \times \mathbb{R}^{3}\right\}$ be a predictable process such that for some $p \geq 2$ and $x \in \mathbb{R}^{3}$,

$$
\mathbb{E}\left(\int_{0}^{t} \int_{\mathbb{R}^{3}} \int_{\mathbb{R}^{3}}|Z(s, x-y) Z(s, x-z)| G(s, d y) G(s, d z) f(y-z) d s\right)^{\frac{p}{2}}<\infty .
$$

Then the measure-valued predictable process $Z(s, y) G(s, x-d y)$ belongs $L^{2}(\Omega \times$ $[0, T] ; U)$ and there exists a positive constant $C_{p}$, depending only on $p$, such that

$$
\begin{aligned}
& \mathbb{E}\left|\int_{0}^{t} \int_{\mathbb{R}^{3}} Z(s, y) G(s, x-d y) W(d s, d y)\right|^{p} \\
& \leq C_{p} \mathbb{E}\left(\int_{0}^{t} \int_{\mathbb{R}^{3}} \int_{\mathbb{R}^{3}} Z(s, x-y) Z(s, x-z) G(s, d y) G(s, d z) f(y-z) d s\right)^{\frac{p}{2}} .
\end{aligned}
$$

If we have

$$
\sup _{(t, x) \in[0, T] \times \mathbb{R}^{3}} \mathbb{E}|Z(t, x)|^{p}<\infty,
$$

then an application of Hölder inequality yields 


$$
\begin{aligned}
& \mathbb{E}\left|\int_{0}^{t} \int_{\mathbb{R}^{3}} Z(s, y) G(s, x-d y) W(d s, d y)\right|^{p} \\
\leq & C_{p} \int_{0}^{t} d s\left(\sup _{x \in \mathbb{R}^{3}} \mathbb{E}|Z(s, x)|^{p}\right)\left(\iint_{\mathbb{R}^{3}} f(y-z) G(s, d y) G(s, d z)\right)^{\frac{p}{2}} .
\end{aligned}
$$

By Lemma 6.4, the above inequality can also be written as

$$
\begin{aligned}
& \mathbb{E}\left|\int_{0}^{t} \int_{\mathbb{R}^{3}} Z(s, y) G(s, x-d y) W(d s, d y)\right|^{p} \\
\leq & C_{p} \int_{0}^{t} d s\left(\sup _{x \in \mathbb{R}^{3}} \mathbb{E}|Z(s, x)|^{p}\right)\left(\int_{|x| \leq 2 s} \frac{f(x)}{|x|} d x\right)^{\frac{p}{2}} .
\end{aligned}
$$

Using the above notion of stochastic integral one can introduce the following definition:

Definition 2.3 A real-valued predictable stochastic process $u=\{u(t, x), 0 \leq t \leq$ $\left.T, x \in \mathbb{R}^{3}\right\}$ is a mild random-field solution of (1.1) if for all $t \in(0, T], x \in \mathbb{R}^{3}$,

$$
\begin{aligned}
u(t, x)= & \frac{d}{d t}\left(G(t) * v_{0}\right)(x)+\left(G(t) * \bar{v}_{0}\right)(x) \\
& +\int_{0}^{t} \int_{\mathbb{R}^{3}} G(t-s, x-d y) \sigma(s, y, u(s, y)) W(d s, d y) \\
& +\int_{0}^{t} G(t-s) *(b(s, \cdot, u(s, \cdot)))(x) d s \quad \text { a.s. }
\end{aligned}
$$

Consider the following condition.

(H) The coefficients $\sigma$ and $b$ satisfy

$$
\begin{aligned}
|\sigma(t, x, u)-\sigma(t, y, v)| & \leq C(|x-y|+|u-v|) \\
|\sigma(s, x, u)| & \leq C(1+|u|)
\end{aligned}
$$

and

$$
\begin{aligned}
|b(t, x, u)-b(t, y, v)| & \leq C(|x-y|+|u-v|) \\
|b(s, x, u)| & \leq C(1+|u|)
\end{aligned}
$$

for any $x, y \in \mathbb{R}^{3}, s, t \in[0, T]$ and $u, v \in \mathbb{R}$. 
Then one can prove the existence and uniqueness of the solution to (1.1) in exactly the same way as in [4, Theorem 4.3].

Theorem 2.4 Suppose the condition (2.5) holds, and $\sigma, b$ satisfy the condition $(\boldsymbol{H})$. Let $v_{0} \in C^{1}\left(\mathbb{R}^{3}\right)$ such that $v_{0}$ and $\nabla v_{0}$ are bounded and $\bar{v}_{0}$ is bounded and continuous. Then there exists a unique mild random-field solution $u$ to (1.1) such that for all $p \geq 1$,

$$
\sup _{(t, x) \in[0, T] \times \mathbb{R}^{3}} \mathbb{E}|u(t, x)|^{p}<\infty
$$

Along the paper, $C$ will denote a generic constant which may change from line to line.

\section{Hölder continuity in the space variable}

In this section we will prove Theorems 3.1 and 3.2 which are the main results on the Hölder continuity of the solution of Eq. (1.1) in the space variable.

Theorem 3.1 Let $u$ be the solution to Eq. (1.1). Assume the following conditions.

(a) The coefficients $\sigma$ and $b$ satisfy condition $(\boldsymbol{H})$.

(b) $v_{0} \in C^{2}\left(\mathbb{R}^{3}\right), v_{0}, \nabla v_{0}$ and $\bar{v}_{0}$ are bounded and $\Delta v_{0}$ and $\bar{v}_{0}$ are Hölder continuous of orders $\gamma_{1}$ and $\gamma_{2}$ respectively, $\gamma_{1}, \gamma_{2} \in(0,1]$.

(c) The function $f$ satisfies condition (2.5) and for some $\gamma \in(0,1]$ and $\gamma^{\prime} \in(0,2]$ we have for all $w \in \mathbb{R}^{3}$ such that $|w| \leq 1$

$$
\int_{|z| \leq 2 T} \frac{|f(z+w)-f(z)|}{|z|} d z \leq C|w|^{\gamma}
$$

and

$$
\int_{|z| \leq 2 T} \frac{|f(z+w)+f(z-w)-2 f(z)|}{|z|} d z \leq C|w|^{\gamma^{\prime}}
$$

Set $\kappa_{1}=\min \left(\gamma_{1}, \gamma_{2}, \gamma, \frac{\gamma^{\prime}}{2}\right)$. Then for any $q \geq 2$, there exists a constant $C$ such that

$$
\sup _{t \in[0, T]} \mathbb{E}|u(t, x)-u(t, y)|^{q} \leq C|x-y|^{q \kappa_{1}}
$$

for any $x, y \in \mathbb{R}^{3}$.

Proof It suffices to assume that $|x-y| \leq 1$. Set $x-y=w$. Fix $q \geq 2$. Then we have

$$
\mathbb{E}|u(t, x)-u(t, y)|^{q} \leq C\left\{\mathbb{E} \mid \int_{0}^{t} \int_{\mathbb{R}^{3}} G(t-s, x-d z) \sigma(s, z, u(s, z)) W(d s, d z)\right.
$$




$$
\begin{aligned}
& -\left.\int_{0}^{t} \int_{\mathbb{R}^{3}} G(t-s, y-d z) \sigma(s, z, u(s, z)) W(d s, d z)\right|^{q} \\
& +\mathbb{E} \mid \int_{0}^{t} G(t-s) * b(s, \cdot, u(s, \cdot))(x) d s \\
& -\left.\int_{0}^{t} G(t-s) * b(s, \cdot, u(s, \cdot))(y) d s\right|^{q} \\
& +\left|\frac{d}{d t}\left(G(t) * v_{0}\right)(x)-\frac{d}{d t}\left(G(t) * v_{0}\right)(y)\right|^{q} \\
& \left.+\left|\left(G(t) * \bar{v}_{0}\right)(x)-\left(G(t) * \bar{v}_{0}\right)(y)\right|^{q}\right\} \\
& :=C\left(I_{1}+I_{2}+I_{3}+I_{4}\right) .
\end{aligned}
$$

For $I_{4}$, since $\bar{v}_{0}$ is Hölder continuous with exponent $\gamma_{2}$ we get

$$
\begin{aligned}
\left|G(t) * \bar{v}_{0}(x)-G(t) * \bar{v}_{0}(y)\right|^{q} & \leq\left|\int_{\mathbb{R}^{3}} G(t, d z)\right| \bar{v}_{0}(x-z)-\left.\bar{v}_{0}(y-z)\right|^{q} \\
& \leq C|w|^{\gamma_{2} q}\left|\int_{\mathbb{R}^{3}} G(t, d z)\right|^{q} \leq C|w|^{\gamma_{2} q}
\end{aligned}
$$

For $I_{3}$, we use the identity (see, for instance, [7])

$$
\frac{d}{d t} G(t) * v_{0}(x)=\frac{1}{t}\left(v_{0} * G(t)\right)(x)+\frac{1}{4 \pi} \int_{|y|<1}\left(\Delta v_{0}\right)(x+t y) d y
$$

Then, since $\Delta v_{0}$ is Hölder continuous with exponent $\gamma_{1}$, we get

$$
\begin{aligned}
\left|\frac{d}{d t}\left(G(t) * v_{0}\right)(x)-\frac{d}{d t}\left(G(t) * v_{0}\right)(y)\right|^{q} \leq & \frac{C}{t^{q}}\left|\int_{\mathbb{R}^{3}} G(t, d z)\left(v_{0}(x-z)-v_{0}(y-z)\right)\right|^{q} \\
& +C\left|\int_{|z|<1}\left(\Delta v_{0}(x+t z)-\Delta v_{0}(y+t z)\right) d z\right|^{q} \\
\leq & C|w|^{\gamma_{1} q} .
\end{aligned}
$$


For $I_{2}$, we use the Lipschitz condition on $b$ and Hölder's inequality to get

$$
\begin{aligned}
& I_{2}=\mathbb{E} \mid \int_{0} \int_{\mathbb{R}^{3}} G(t-s, d z) b(s, x-z, u(s, x-z)) d s \\
&-\left.\int_{0}^{t} \int_{\mathbb{R}^{3}} G(t-s, d z) b(s, y-z, u(s, y-z)) d s\right|^{q} \\
& \leq\left.C \mathbb{E}_{0}^{t} \int_{0} G(t-s, d z)(|w|+|u(s, x-z)-u(s, y-z)|) d s\right)_{\mathbb{R}^{3}}^{q} \\
& \leq\left.C \mathbb{E}^{t} \int_{0}^{t} \int_{\mathbb{R}^{3}} G(t-s, d z) d s\right)^{q-1}\left(\int_{\mathbb{R}^{3}}^{t} G(t-s, d z)|w|^{q} d s\right. \\
&\left.+\int_{0}^{t} \int_{\mathbb{R}^{3}} G(t-s, d z)|u(s, x-z)-u(s, y-z)|^{q} d s\right) \\
& \leq C|w|^{q}+C \int_{0}^{t} d s \quad z_{1}-z_{2}=w \\
& \mathbb{E}\left|u\left(s, z_{1}\right)-u\left(s, z_{2}\right)\right|^{q} .
\end{aligned}
$$

For $I_{1}$, we apply the Burkholder's inequality of Lemma 2.2 to get

$$
\begin{aligned}
& I_{1} \leq C \mathbb{E} \mid \int_{0} \int_{\mathbb{R}^{3}} \int_{\mathbb{R}^{3}} \sigma(s, \xi, u(s, \xi)) f(\xi-\eta) \sigma(s, \eta, u(s, \eta)) \\
& \times(G(t-s, x-d \xi)-G(t-s, y-d \xi))(G(t-s, x-d \eta) \\
&\quad-G(t-s, y-d \eta))\left.d s\right|^{\frac{q}{2}} \\
&:=C \mathbb{E}|Q|^{\frac{q}{2}}
\end{aligned}
$$

The main idea to estimate the above quantity is to transfer the increments of $G$ to increments of $f$ and $\sigma$. We introduce the following notation

$$
\begin{aligned}
\Sigma_{x}(s, \xi) & =\sigma(s, x-\xi, u(s, x-\xi)) \\
\Sigma_{x, y}(s, \xi) & =\sigma(s, x-\xi, u(s, x-\xi))-\sigma(s, y-\xi, u(s, y-\xi)) .
\end{aligned}
$$


Define

$$
\begin{aligned}
& h_{1}=f(\eta-\xi) \Sigma_{x, y}(s, \xi) \Sigma_{x, y}(s, \eta), \\
& h_{2}=(f(\eta-\xi+w)-f(\eta-\xi)) \Sigma_{x}(s, \xi) \Sigma_{x, y}(s, \eta), \\
& h_{3}=(f(\eta-\xi-w)-f(\eta-\xi)) \Sigma_{x}(s, \eta) \Sigma_{x, y}(s, \xi), \\
& h_{4}=(2 f(\eta-\xi)-f(\eta-\xi+w)-f(\eta-\xi-w)) \Sigma_{x}(s, \xi) \Sigma_{x}(s, \eta) .
\end{aligned}
$$

and

$$
Q_{i}=\int_{0}^{t} \int_{\mathbb{R}^{3}} \int_{\mathbb{R}^{3}} G(t-s, d \xi) G(t-s, d \eta) h_{i} d s, \quad i=1,2,3,4 .
$$

Then by direct calculation, we can verify that $Q=\sum_{i=1}^{4} Q_{i}$. To estimate $\mathbb{E}|Q|^{\frac{q}{2}}$, we need to estimate $\mathbb{E}\left|Q_{i}\right|^{\frac{q}{2}}$ for $i=1, \ldots, 4$. For $\mathbb{E}\left|Q_{1}\right|^{\frac{q}{2}}$, by the assumptions on $\sigma$, using Hölder's inequality and identities (2.7) we have

$$
\begin{aligned}
& \mathbb{E}\left|Q_{1}\right|^{\frac{q}{2}}=\mathbb{E} \int_{0}^{t} d s \int_{\mathbb{R}^{3}} \int_{\mathbb{R}^{3}} G(t-s, d \xi) G(t-s, d \eta) f(\eta-\xi) \\
& \times(\sigma(s, x-\xi, u(s, x-\xi))-\sigma(s, y-\xi, u(s, y-\xi))) \\
& \times\left.(\sigma(s, x-\eta, u(s, x-\eta))-\sigma(s, y-\eta, u(s, y-\eta)))\right|^{\frac{q}{2}} \\
& \leq C \mathbb{E} \mid \int_{0}^{t} d s \int_{\mathbb{R}^{3}} \int_{\mathbb{R}^{3}} G(t-s, d \xi) G(t-s, d \eta) f(\eta-\xi) \\
& \times(|w|+|u(s, x-\xi)-u(s, y-\xi)|) \\
& \times\left.(|w|+|u(s, x-\eta)-u(s, y-\eta)|)\right|^{\frac{q}{2}} \\
& \leq C \int_{0}^{t} d s\left(\int_{\mathbb{R}^{3}} \int_{\mathbb{R}^{3}} G(t-s, d \xi) G(t-s, d \eta) f(\eta-\xi)\right)^{\frac{q}{2}} \\
& \times\left(|w|^{q}+\sup _{z_{1}-z_{2}=w} \mathbb{E}\left|u\left(s, z_{1}\right)-u\left(s, z_{2}\right)\right|^{q}\right) \\
& \leq C \int_{0}^{t} d s\left(\int_{|z| \leq 2 T} \frac{f(z)}{|z|} d z\right)^{\frac{q}{2}}\left(|w|^{q}+\sup _{z_{1}-z_{2}=w} \mathbb{E}\left|u\left(s, z_{1}\right)-u\left(s, z_{2}\right)\right|^{q}\right) .
\end{aligned}
$$


By the condition (2.5), we get

$$
\mathbb{E}\left|Q_{1}\right|^{\frac{q}{2}} \leq C|w|^{q}+C \int_{0}^{t} d s \sup _{z_{1}-z_{2}=w} \mathbb{E}\left|u\left(s, z_{1}\right)-u\left(s, z_{2}\right)\right|^{q} .
$$

For $\mathbb{E}\left|Q_{2}\right|^{\frac{q}{2}}$, we write $f(\eta-\xi+w)-f(\eta-\xi)=\Phi_{1}(\eta-\xi, w)$ and using the inequality $a b \leq \frac{a^{2}+b^{2}}{2}$ we obtain

$$
\begin{aligned}
\mathbb{E}\left|Q_{2}\right|^{\frac{q}{2}} \leq & C \mathbb{E}\left(\int_{0}^{t} \int_{\mathbb{R}^{3}} \int_{\mathbb{R}^{3}}\left|\Phi_{1}(\eta-\xi, w)\right|\left|\Sigma_{x}(s, \xi)\right|\left|\Sigma_{x, y}(s, \eta)\right| G(t-s, d \xi) G(t-s, d \eta) d s\right)^{\frac{q}{2}} \\
\leq & C \mathbb{E}\left(\int_{0}^{t} \int_{\mathbb{R}^{3}} \int_{\mathbb{R}^{3}}|w|^{\gamma}\left|\Phi_{1}(\eta-\xi, w)\right|\left|\Sigma_{x}(s, \xi)\right|^{2} G(t-s, d \xi) G(t-s, d \eta) d s\right)^{\frac{q}{2}} \\
& +C \mathbb{E}\left(\int_{0}^{t} \int_{\mathbb{R}^{3}} \int_{\mathbb{R}^{3}} \frac{\left|\Phi_{1}(\eta-\xi, w)\right|}{|w|^{\gamma}}\left|\Sigma_{x, y}(s, \eta)\right|^{2} G(t-s, d \xi) G(t-s, d \eta) d s\right)^{\frac{q}{2}} \\
:=C\left(Q_{2,1}+Q_{2,2}\right) . &
\end{aligned}
$$

Applying condition (3.1), identities (2.7) and Hölder's inequality yields

$$
\begin{aligned}
Q_{2,1} \leq & |w|^{\frac{q \gamma}{2}}\left(\int_{0}^{t} \int_{\mathbb{R}^{3}} \int_{\mathbb{R}^{3}}\left|\Phi_{1}(\eta-\xi, w)\right| G(t-s, d \xi) G(t-s, d \eta) d s\right)^{\frac{q}{2}} \\
& \times \sup _{s, \xi} \mathbb{E}\left|\Sigma_{x}(s, \xi)\right|^{q} \\
\leq & C|w|^{\frac{q \gamma}{2}}\left(\int_{0}^{t} \int_{|z| \leq 2 T} \frac{|f(z+w)-f(z)|}{|z|} d z d s\right)^{\frac{q}{2}} \leq C|w|^{q \gamma} .
\end{aligned}
$$

For the second term we obtain

$$
\begin{aligned}
Q_{2,2} \leq C|w|^{-\frac{q \gamma}{2}} \mathbb{E}\left(\int_{0}^{t} \int_{\mathbb{R}^{3}} \int_{\mathbb{R}^{3}}\left|\Phi_{1}(\eta-\xi, w)\right|\left(|w|^{2}+|u(s, x-\xi)-u(s, y-\xi)|^{2}\right)\right. \\
\times G(t-s, d \xi) G(t-s, d \eta) d s)^{\frac{q}{2}} \\
\leq C|w|^{q-\frac{q \gamma}{2}}\left(\int_{0}^{t} \int_{|z| \leq 2 T} \frac{|f(z+w)-f(z)|}{|z|} d z d s\right)^{\frac{q}{2}}
\end{aligned}
$$




$$
\begin{gathered}
+C|w|^{-\frac{q \gamma}{2}}\left(\int_{|z| \leq 2 T} \frac{|f(z+w)-f(z)|}{|z|} d z\right)^{\frac{q}{2}} \\
\times \int_{0}^{t} \sup _{z_{2}-z_{1}=w} \mathbb{E}\left|u\left(s, z_{1}\right)-u\left(s, z_{2}\right)\right|^{q} d s \\
\leq C|w|^{q}+C \int_{0}^{t} \sup _{z_{2}-z_{1}=w} \mathbb{E}\left|u\left(s, z_{1}\right)-u\left(s, z_{2}\right)\right|^{q} d s .
\end{gathered}
$$

So we conclude that

$$
\mathbb{E}\left|Q_{2}\right|^{\frac{q}{2}} \leq C|w|^{q \gamma}+C \int_{0}^{t} \sup _{z_{2}-z_{1}=w} \mathbb{E}\left|u\left(s, z_{1}\right)-u\left(s, z_{2}\right)\right|^{q} d s .
$$

The term $\mathbb{E}\left|Q_{3}\right|^{\frac{q}{2}}$ can be treated in the same way and we have

$$
\mathbb{E}\left|Q_{3}\right|^{\frac{q}{2}} \leq C|w|^{q \gamma}+C \int_{0}^{t} \sup _{z_{2}-z_{1}=w} \mathbb{E}\left|u\left(s, z_{1}\right)-u\left(s, z_{2}\right)\right|^{q} d s .
$$

For $\mathbb{E}\left|Q_{4}\right|^{\frac{q}{2}}$, we set $\Phi_{2}(\eta-\xi, w)=f(\eta-\xi+w)+f(\eta-\xi-w)-2 f(\eta-\xi)$, and using the assumption on $\sigma$, condition (3.2), Hölder's inequality and the moments estimate (2.15), we have

$$
\begin{aligned}
\mathbb{E}\left|Q_{4}\right|^{\frac{q}{2}}= & \mathbb{E}\left(\int_{0}^{t} \int_{\mathbb{R}^{3}} \int_{\mathbb{R}^{3}}\left|\Phi_{2}(\eta-\xi, w)\right|\left|\Sigma_{x}(s, \xi) \Sigma_{x}(s, \eta)\right| G(t-s, d \xi) G(t-s, d \eta) d s\right)^{\frac{q}{2}} \\
\leq & \left(\int_{0}^{t} \int_{\mathbb{R}^{3}} \int_{\mathbb{R}^{3}}\left|\Phi_{2}(\eta-\xi, w)\right| G(t-s, d \xi) G(t-s, d \eta) d s\right)^{\frac{q}{2}} \\
& \times \sup _{s, \xi, \eta} \mathbb{E}\left(\left|\Sigma_{x}(s, \xi)\right|\left|\Sigma_{x}(s, \eta)\right|\right)^{\frac{q}{2}} \\
\leq & C\left(\int_{0}^{t} \int_{|z| \leq 2 T} \frac{|f(z+w)+f(z-w)-2 f(z)|}{|z|} d z d s\right)^{\frac{q}{2}} \leq C|w|^{\frac{q \gamma^{\prime}}{2}} .
\end{aligned}
$$

Combining the above expression with (3.14), (3.15) and (3.16), we can write

$$
I_{1} \leq C\left(|w|^{\gamma q}+|w|^{\frac{\gamma^{\prime} q}{2}}\right)+C \int_{0}^{t} \sup _{z_{2}-z_{1}=w} \mathbb{E}\left|u\left(s, z_{1}\right)-u\left(s, z_{2}\right)\right|^{q} d s
$$


The estimates for $I_{i}, i=1,2,3,4$, lead to

$$
\begin{aligned}
& \sup _{z_{1}-z_{2}=w} \mathbb{E}\left|u\left(t, z_{1}\right)-u\left(t, z_{2}\right)\right|^{q} \\
& \leq C|w|^{q \min \left(\gamma_{1}, \gamma_{2}, \gamma, \frac{\gamma^{\prime}}{2}\right)}+C \int_{0}^{t} d s \sup _{z_{1}-z_{2}=w}\left|u\left(s, z_{1}\right)-u\left(s, z_{2}\right)\right|^{q} .
\end{aligned}
$$

An application of Gronwall's lemma yields

$$
\mathbb{E}|u(t, x)-u(t, y)|^{q} \leq C|x-y|^{q \min \left(\gamma_{1}, \gamma_{2}, \gamma, \frac{\gamma^{\prime}}{2}\right)}
$$

for any $x$ and $y$ in $\mathbb{R}^{3}$ such that $|x-y| \leq 1$, which completes the proof of the theorem. Notice that, as it can be checked throughout the proof, the generic constant $C$ does not depend on $t \in[0, T]$.

Next we give a theorem which establishes the Hölder continuity in the space variable using the Fourier transform.

Theorem 3.2 Let $u$ be the solution to Eq. (1.1). Assume conditions (a) and (b) in Theorem 3.1. Suppose the following condition:

$\left(c^{\prime}\right)$ For some $\gamma \in(0,1]$, the Fourier transform of the tempered measure $|\zeta|^{2 \gamma} \mu(d \zeta)$ is a nonnegative locally integrable function and

$$
\int_{\mathbb{R}^{3}} \frac{\mu(d \zeta)}{1+|\zeta|^{2-2 \gamma}}<\infty
$$

Set $\kappa_{1}^{\prime}=\min \left(\gamma_{1}, \gamma_{2}, \gamma\right)$. Then for any $q \geq 2$, there exists a constant $C$ such that

$$
\sup _{t \in[0, T]} \mathbb{E}|u(t, x)-u(t, y)|^{q} \leq C|x-y|^{q \kappa_{1}^{\prime}}
$$

for any $x, y \in \mathbb{R}^{3}$.

Proof It suffices to assume that $|x-y| \leq 1$. Set $x-y=w$. Fix $q \geq 2$, as in the proof of Theorem 3.1, we still express $\mathbb{E}|u(t, x)-u(t, y)|^{q}$ as $C\left(I_{1}+I_{2}+I_{3}+I_{4}\right)$, and the estimates for $I_{2}, I_{3}, I_{4}$ are the same as in the proof of Theorem 3.1. For $I_{1}$, use the notation (3.7)-(3.13) and we need to estimate $\mathbb{E}\left|Q_{i}\right|^{\frac{q}{2}}$ for $i=1, \ldots, 4$.

The estimate for $\mathbb{E}\left|Q_{1}\right|^{\frac{q}{2}}$ is the same as in the proof of Theorem 3.1.

For $Q_{2}$ we would like to apply Eq. (6.17) to $\varphi=\Sigma_{x, y}(s, \eta)$ and $\psi=\Sigma_{x}(s, \xi)$. Because these functions are not necessarily bounded we we introduce the truncations

$$
\Sigma_{x}^{k}(s, \xi)=\Sigma_{x}(s, \xi) \mathbf{1}_{\left\{\left|\Sigma_{x}(s, \xi)\right| \leq k\right\}},
$$




$$
\Sigma_{x, y}^{k}(s, \eta)=\Sigma_{x, y}(s, \eta) \mathbf{1}_{\left\{\left|\Sigma_{x, y}(s, \eta)\right| \leq k\right\}}
$$

for any $k>0$. Clearly, as $k$ tends to infinity, $\Sigma_{x}^{k}(s, \xi)$ and $\Sigma_{x, y}^{k}(s, \eta)$ converge pointwise to $\Sigma_{x}(s, \xi)$ and $\Sigma_{x, y}(s, \eta)$, respectively. Set

$$
\begin{aligned}
Q_{2}^{k}= & \int_{0}^{t} d s \int_{\mathbb{R}^{3}} \int_{\mathbb{R}^{3}} G(t-s, d \xi) G(t-s, d \eta)(f(\eta-\xi+w) \\
& -f(\eta-\xi)) \Sigma_{x}^{k}(s, \xi) \Sigma_{x, y}^{k}(s, \eta) .
\end{aligned}
$$

Then Eq. (6.17) yields

$$
Q_{2}^{k}=\int_{0}^{t} d s \int_{\mathbb{R}^{3}} \overline{\mathcal{F}\left(\Sigma_{x}^{k}(s, \cdot) G(t-s)\right)(\zeta)} \mathcal{F}\left(\Sigma_{x, y}^{k}(s, \cdot) G(t-s)\right)(\zeta)\left(e^{-i w \cdot \zeta}-1\right) \mu(d \zeta)
$$

Using the estimate $\left|e^{-i w \cdot \zeta}-1\right| \leq C|w|^{\gamma}|\zeta|^{\gamma}$ for every $0<\gamma \leq 1$, Cauchy-Schwartz's inequality and the inequality $\sqrt{a b} \leq \frac{1}{2}(a+b)$ for any $a, b>0$, we can write

$$
\begin{aligned}
\left|Q_{2}^{k}\right| \leq & \int_{0}^{t} d s \int_{\mathbb{R}^{3}}\left|\mathcal{F}\left(\Sigma_{x}^{k}(s, \cdot) G(t-s)\right)(\zeta)\right|\left|\mathcal{F}\left(\Sigma_{x, y}^{k}(s, \cdot) G(t-s)\right)(\zeta)\right||w|^{\gamma}|\zeta|^{\gamma} \mu(d \zeta) \\
\leq & \int_{0}^{t} d s|w|^{\gamma}\left(\int_{\mathbb{R}^{3}}\left|\mathcal{F}\left(\Sigma_{x}^{k}(s, \cdot) G(t-s)\right)(\zeta)\right|^{2}|\zeta|^{2 \gamma} \mu(d \zeta)\right)^{\frac{1}{2}} \\
& \left.\times \int_{\mathbb{R}^{3}}\left|\mathcal{F}\left(\Sigma_{x, y}^{k}(s, \cdot) G(t-s)\right)(\zeta)\right|^{2} \mu(d \zeta)\right)^{\frac{1}{2}} \\
\leq & \frac{1}{2} \int_{0}^{t} d s|w|^{2 \gamma} \int_{\mathbb{R}^{3}}\left|\mathcal{F}\left(\Sigma_{x}^{k}(s, \cdot) G(t-s)\right)(\zeta)\right|^{2}|\zeta|^{2 \gamma} \mu(d \zeta) \\
& +\frac{1}{2} \int_{0}^{t} d s \int_{\mathbb{R}^{3}}\left|\mathcal{F}\left(\Sigma_{x, y}^{k}(s, \cdot) G(t-s)\right)(\zeta)\right|^{2} \mu(d \zeta) \\
= & \frac{1}{2}|w|^{2 \gamma} \int_{0}^{t} d s \int_{\mathbb{R}^{3}} d \eta g(\eta)\left[\left(\Sigma_{x}^{k}(s, \cdot) G(t-s)\right) *\left(\Sigma_{x}^{k}(s, \cdot) G(t-s)\right)\right](\eta)
\end{aligned}
$$




$$
\begin{aligned}
& \left.\quad+\frac{1}{2} \int_{0}^{t} d s \int_{\mathbb{R}^{3}} d \eta f(\eta)\left[\left(\Sigma_{x, y}^{k}(s, \cdot) G(t-s)\right) *\left(\Sigma_{x, y}^{k} \widetilde{(s, \cdot) G(t}-s\right)\right)\right](\eta) \\
& :=Q_{2,1}^{k}+Q_{2,2}^{k},
\end{aligned}
$$

where $g$ is the Fourier transform of the measure $|\cdot|^{2 \gamma} \mu$, which by our hypothesis is a nonnegative locally integrable function. In the above formula, for any measure $v, \widetilde{v}$ denotes the measure $\widetilde{v}(A)=v(-A)$. Treating $g(\eta) G(t-s) * G(t-s)(\eta) d \eta$ as a new measure, and using Minkowski's inequality, we get

$$
\begin{aligned}
\mathbb{E}\left|Q_{2,1}^{k}\right|^{\frac{q}{2}} \leq C|w|^{q \gamma} \int_{0}^{t} d s\left(\int_{\mathbb{R}^{3}} d \eta g(\eta)[G(t-s) * G(t-s)](\eta)\right)^{\frac{q}{2}} \\
\times \sup _{0 \leq s \leq t, \xi, \eta \in \mathbb{R}^{3}} \mathbb{E}\left|\Sigma_{x}(s, \xi) \Sigma_{x}(s, \xi+\eta)\right|^{\frac{q}{2}} \\
\leq C|w|^{q \gamma} \int_{0}^{t} d s\left(\int_{\mathbb{R}^{3}}|\zeta|^{2 \gamma} \mu(d \zeta) \mathcal{F}(G(t-s) * G(t-s))(\zeta)\right)^{\frac{q}{2}} \\
\leq C|w|^{q \gamma}\left(\int_{\mathbb{R}^{3}} \mu(d \zeta) \frac{|\zeta|^{2 \gamma}}{1+|\zeta|^{2}}\right)^{\frac{q}{2}} \leq C|w|^{q \gamma}
\end{aligned}
$$

where we have used the moments estimate (2.15), Eq. (2.4), the fact that $\left(\frac{\sin (s|\xi|)}{|\xi|}\right)^{2} \leq$ $\frac{C}{1+|\xi|^{2}}$, when $s \in[0, T]$ and the inequality $\left|\Sigma_{x}^{k}(s, \xi)\right| \leq\left|\Sigma_{x}(s, \xi)\right|$. Therefore,

$$
\begin{aligned}
\mathbb{E}\left|Q_{2}^{k}\right|^{\frac{q}{2}} \leq C|w|^{q \gamma} \\
+C \mathbb{E} \int_{0}^{t} d s\left(\int_{\mathbb{R}^{3}} d \eta f(\eta)\left[\left(\Sigma_{x, y}^{k}(s, \cdot) G(t-s)\right) *\left(\Sigma_{x, y}^{k}(\widetilde{s, \cdot) G(t}-s)\right)\right](\eta)\right)^{\frac{q}{2}} .
\end{aligned}
$$

Applying the dominated convergence theorem we can show that in the above inequality, as $k$ goes to infinity, the left-hand side converges to $\mathbb{E}\left|Q_{2}\right|^{\frac{q}{2}}$ and the expectation on the right-hand side converges to

$$
\mathbb{E} \int_{0}^{t} d s\left(\int_{\mathbb{R}^{3}} f(\eta) d \eta\left[\left(\Sigma_{x, y}(s, \cdot) G(t-s)\right) *\left(\Sigma_{x, y}(\widetilde{s, \cdot) G(t}-s)\right)\right](\eta)\right)^{\frac{q}{2}} .
$$


From the expression for $\Sigma_{x, y}(s, \xi)$ and using Minkowski's inequality, we have

$$
\begin{aligned}
\mathbb{E}\left|Q_{2}\right|^{\frac{q}{2}} \leq C|w|^{q \gamma}+C \int_{0}^{t} d s\left(\int_{\mathbb{R}^{3}} d \eta f(\eta)(G(t-s) * G(t-s))(\eta)\right)^{\frac{q}{2}} \\
\quad \times \sup _{\eta \in \mathbb{R}^{3}} \mathbb{E}\left[|\sigma(s, x-\eta, u(s, x-\eta))-\sigma(s, y-\eta, u(s, y-\eta))|^{q}\right] \\
\leq C|w|^{q \gamma}+C \int_{0}^{t} d s\left(\int_{\mathbb{R}^{3}} \frac{d \mu(\zeta)}{1+|\zeta|^{2}}\right)^{\frac{q}{2}}\left(|w|^{q}+\sup _{z_{1}-z_{2}=w} \mathbb{E}\left|u\left(s, z_{1}\right)-u\left(s, z_{2}\right)\right|^{q}\right) \\
\leq C\left(|w|^{q \gamma}+|w|^{q}+\int_{0}^{t} d s \sup _{z_{1}-z_{2}=w} \mathbb{E}\left|u\left(s, z_{1}\right)-u\left(s, z_{2}\right)\right|^{q}\right) .
\end{aligned}
$$

The same estimate holds for $\mathbb{E}\left|Q_{3}\right|^{\frac{q}{2}}$.

Consider now the term $Q_{4}$. We use the truncation argument as in the estimation for $\mathbb{E}\left|Q_{2}\right|^{\frac{q}{2}}$ and we set

$$
\begin{aligned}
Q_{4}^{k}= & \int_{0}^{t} d s \int_{\mathbb{R}^{3}} \int_{\mathbb{R}^{3}} G(t-s, d \xi) G(t-s, d \eta)(2 f(\eta-\xi) \\
& \quad-f(\eta-\xi+w)-f(\eta-\xi-w)) \times \Sigma_{x}^{k}(s, \xi) \Sigma_{x}^{k}(s, \eta) .
\end{aligned}
$$

Then, Eq. (6.17) implies

$$
\begin{aligned}
Q_{4}^{k} & =\int_{0}^{t} d s \int_{\mathbb{R}^{3}} \mu(d \zeta)(1-\cos (w \cdot \zeta))\left|\left(\mathcal{F}\left(\Sigma_{x}^{k}(s, \cdot) G(t-s)\right)\right)(\zeta)\right|^{2} \\
& \leq 2|w|^{2 \gamma} \int_{0}^{t} d s \int_{\mathbb{R}^{3}} \mu(d \zeta)|\zeta|^{2 \gamma}\left|\left(\mathcal{F}\left(\Sigma_{x}^{k}(s, \cdot) G(t-s)\right)\right)(\zeta)\right|^{2} \\
& =2|w|^{2 \gamma} \int_{0}^{t} d s \int_{\mathbb{R}^{3}} d \eta g(\eta)\left(\left(\Sigma_{x}^{k}(s, \cdot) G(t-s)\right) *\left(\Sigma_{x}^{k}(\widehat{s, \cdot) G(t}-s)\right)\right)(\eta) .
\end{aligned}
$$

Then we can use the same argument as before, to conclude that

$$
\mathbb{E}\left|Q_{4}\right|^{\frac{q}{2}} \leq C|w|^{q \gamma}
$$


Combining the moment estimates for $E\left|Q_{i}\right|^{\frac{q}{2}}, i=1,2,3,4$, since $|w| \leq 1$ and $0<\gamma \leq 1$, we have

$$
I_{1} \leq C|w|^{q \gamma}+C \int_{0}^{t} d s \sup _{z_{1}-z_{2}=w} \mathbb{E}\left|u\left(s, z_{1}\right)-u\left(s, z_{2}\right)\right|^{q} .
$$

Finally, the estimates for $I_{i}, i=1,2,3,4$, allow us to write

$$
\begin{aligned}
& \sup _{z_{1}-z_{2}=x-y} \mathbb{E}\left|u\left(t, z_{1}\right)-u\left(t, z_{2}\right)\right|^{q} \\
& \leq C|x-y|^{q \min \left(\gamma_{1}, \gamma_{2}, \gamma\right)}+C \int_{0}^{t} d s \sup _{z_{1}-z_{2}=x-y} \mathbb{E}\left|u\left(s, z_{1}\right)-u\left(s, z_{2}\right)\right|^{q} .
\end{aligned}
$$

An application of Gronwall's lemma yields

$$
\mathbb{E}|u(t, x)-u(t, y)|^{q} \leq C|x-y|^{q \min \left(\gamma_{1}, \gamma_{2}, \gamma\right)}
$$

for any $x$ and $y$ in $\mathbb{R}^{3}$ such that $|x-y| \leq 1$, which completes the proof of the theorem. Notice that, as it can be checked throughout the proof, the generic constant $C$ does not depend on $t \in[0, T]$.

Under the assumptions of Theorem 3.1 or Theorem 3.2, applying Kolmogorov's continuity criterion, for any fixed $t \in[0, T]$, we deduce the existence of a locally Hölder continuous version for the process $\left\{u(t, x), x \in \mathbb{R}^{3}\right\}$ with exponent $\kappa>0$ where $\kappa<\kappa_{1}$. Namely, for any $t \in[0, T]$ and any compact rectangle $I \subset \mathbb{R}^{3}$, there exists a random variable $K_{\kappa, t, I}$ such that

$$
|u(t, x)-u(t, y)| \leq K_{\kappa, t, I}|x-y|^{\kappa}
$$

for and $x, y \in I$.

\section{Hölder continuity in space and time variables}

In this section we obtain a result on the Hölder continuity of the solution of Eq. (1.1) in both the space and time variables. Let $S^{2}$ denote the unit sphere in $\mathbb{R}^{3}$ and $\sigma(d \xi)$ the uniform measure on it. We have the following result.

Theorem 4.1 Let $u$ be the solution to Eq. (1.1). Assume conditions (a) and (b) in Theorem 3.1. Suppose the following conditions hold.

(1) For some $0<v \leq 1, \int_{|z| \leq h} \frac{f(z)}{|z|} d z \leq C h^{v}$ for any $0<h \leq 2 T$.

(2) For some $0<\kappa_{1} \leq 1$ and for any $q \geq 2$ and $t \in(0, T]$, we have

$$
\mathbb{E}|u(t, x)-u(t, y)|^{q} \leq C|x-y|^{q \kappa_{1}} .
$$


(3) Let $\xi$ and $\eta$ be unit vectors in $\mathbb{R}^{3}$ and $0<h \leq 1$. We have

$$
\iint_{0}^{T} \int_{S^{2} S^{2}}|f(s(\xi+\eta)+h(\xi+\eta))-f(s(\xi+\eta)+h \eta)| s \sigma(d \xi) \sigma(d \eta) d s \leq C h^{\rho_{1}}
$$

for some $\rho_{1} \in(0,1]$, and

$$
\begin{aligned}
& \int_{0}^{T} \int_{S^{2}} \int_{S^{2}} \mid f(s(\xi+\eta)+h(\xi+\eta))-f(s(\xi+\eta)+h \xi) \\
& \quad-f(s(\xi+\eta)+h \eta)+f(s(\xi+\eta)) \mid s^{2} \sigma(d \xi) \sigma(d \eta) d s \leq C h^{\rho_{2}}
\end{aligned}
$$

for some $\rho_{2} \in(0,2]$.

Set $\kappa_{2}=\min \left(\gamma_{1}, \gamma_{2}, \kappa_{1}, \frac{v+1}{2}, \frac{\rho_{1}+\kappa_{1}}{2}, \frac{\rho_{2}}{2}\right)$. Then for any $q \geq 2$, there exists a constant C such that

$$
\sup _{x \in \mathbb{R}^{3}} \mathbb{E}|u(\bar{t}, x)-u(t, x)|^{q} \leq C|\bar{t}-t|^{q \kappa_{2}}
$$

for any $t, \bar{t} \in[0, T]$.

Proof Fix $x \in \mathbb{R}^{3}$ and $q \in[2, \infty)$. For all $0 \leq t \leq \bar{t} \leq T$ we can write, by Definition 2.3,

$$
\mathbb{E}|u(t, x)-u(\bar{t}, x)|^{q} \leq C \sum_{i=1}^{4} T_{i}
$$

where

$$
\begin{aligned}
& T_{1}=\left|\left(\frac{d}{d t} G(t) * v_{0}\right)(x)-\left(\frac{d}{d t} G(\bar{t}) * v_{0}\right)(x)\right|^{q}, \\
& T_{2}=\left|\left(G(t) * \bar{v}_{0}\right)(x)-\left(G(\bar{t}) * \bar{v}_{0}\right)(x)\right|^{q}, \\
& T_{3}=\mathbb{E} \mid \int_{0}^{t} d s \int_{\mathbb{R}^{3}} G(t-s, d y) b(s, x-y, u(s, x-y)) \\
& \quad-\left.\int_{0}^{\bar{t}} d s \int_{\mathbb{R}^{3}} G(\bar{t}-s, d y) b(s, x-y, u(s, x-y))\right|^{q},
\end{aligned}
$$




$$
\begin{aligned}
T_{4}=\mathbb{E} & \mid \int_{0}^{t} \int_{\mathbb{R}^{3}} G(t-s, x-d y) \sigma(s, y, u(s, y)) W(d s, d y) \\
& -\left.\int_{0}^{\bar{t}} \int_{\mathbb{R}^{3}} G(\bar{t}-s, x-d y) \sigma(s, y, u(s, y)) W(d s, d y)\right|^{q} .
\end{aligned}
$$

Let $\gamma^{\prime}=\min \left(\gamma_{1}, \gamma_{2}\right)$. By our assumptions on $\Delta v_{0}$ and $\bar{v}_{0}$ and by Lemma 4.9 in [5], we have

$$
T_{1}+T_{2} \leq C|t-\bar{t}|^{q \gamma^{\prime}}
$$

Notice that Lemma 4.9 in [5] assumes that $x$ belongs to a bounded set in $\mathbb{R}^{3}$, but from the proof it is easy to see that the constant $C$ does not depend on $x$.

The term $T_{3}$ is bounded by

$$
T_{3} \leq C\left(T_{3,1}+T_{3,2}\right)
$$

where

$$
\begin{aligned}
& T_{3,1}=\mathbb{E}\left|\int_{t}^{\bar{t}} d s \int_{\mathbb{R}^{3}} G(\bar{t}-s, d y) b(s, x-y, u(s, x-y))\right|^{q}, \\
& T_{3,2}=\mathbb{E}\left|\int_{0}^{t} d s \int_{\mathbb{R}^{3}}(G(t-s, d y)-G(\bar{t}-s, d y)) b(s, x-y, u(s, x-y))\right|^{q} .
\end{aligned}
$$

Hölder's inequality, the linear growth of $b$ and the moments estimate (2.15) imply

$$
\begin{aligned}
& T_{3,1} \leq C\left(\int_{t}^{\bar{t}} d s \int_{\mathbb{R}^{3}} G(\bar{t}-s, d y)\right)^{q-1} \\
& \times\left(\int_{t}^{\bar{t}} d s \int_{\mathbb{R}^{3}} G(\bar{t}-s, d y) \sup _{0 \leq s \leq T} \sup _{x \in \mathbb{R}^{3}}\left(1+\mathbb{E}|u(s, x)|^{q}\right)\right) \\
& \leq C(\bar{t}-t)^{q} .
\end{aligned}
$$

For $T_{3,2}$, we split the integral into a difference of two integrals and then we apply the change of variables $\frac{y}{t-s} \rightarrow y$ and $\frac{y}{\bar{t}-s} \rightarrow y$, respectively. In this way, taking into account that $G(t, d y)=t^{-2} G\left(1, t^{-1} d y\right)$, we get 


$$
\begin{aligned}
T_{3,2}=\mathbb{E} & \mid \int_{0}^{t} d s \int_{\mathbb{R}^{3}} G(1, d y) b(s, x-(t-s) y, u(s, x-(t-s) y))(t-s) \\
& -\left.\int_{0}^{t} d s \int_{\mathbb{R}^{3}} G(1, d y) b(s, x-(\bar{t}-s) y, u(s, x-(\bar{t}-s) y))(\bar{t}-s)\right|^{q} .
\end{aligned}
$$

Hence, $T_{3,2} \leq C\left(T_{3,2,1}+T_{3,2,2}\right)$, where

$$
T_{3,2,1}=(\bar{t}-t)^{q} \mathbb{E}\left|\int_{0}^{t} d s \int_{\mathbb{R}^{3}} G(1, d y) b(s, x-(\bar{t}-s) y, u(s, x-(\bar{t}-s) y))\right|^{q}
$$

and

$$
\begin{aligned}
T_{3,2,2}=\mathbb{E} & \left(\int_{0}^{t} d s(t-s) \int_{\mathbb{R}^{3}} G(1, d y) \mid b(s, x-(\bar{t}-s) y, u(s, x-(\bar{t}-s) y))\right. \\
& -b(s, x-(t-s) y, u(s, x-(t-s) y)) \mid)^{q} .
\end{aligned}
$$

By the moments estimate (2.15) and the linear growth of $b$, it follows that

$$
T_{3,2,1} \leq C|\bar{t}-t|^{q}
$$

Moreover, by the Lipschitz property of $b$ and Hölder continuity assumption on the space variable (condition (2) in the theorem), we get

$$
\begin{aligned}
T_{3,2,2} \leq & C \mathbb{E}\left(\int_{0}^{t} d s(t-s) \int_{\mathbb{R}^{3}} G(1, d y)((\bar{t}-t)|y|\right. \\
& +|u(s, x-(\bar{t}-s) y)-u(s, x-(t-s) y)|))^{q} \\
\leq & \left(\int_{0}^{t} d s(t-s) \int_{\mathbb{R}^{3}} G(1, d y)\right)_{0}^{q-1} \int_{0}^{t} d s(t-s) \int_{\mathbb{R}^{3}} G(1, d y)\left((\bar{t}-t)^{q}|y|^{q}\right. \\
& \left.+\sup _{x \in \mathbb{R}^{3}} \mathbb{E}|u(s, x-(\bar{t}-s) y)-u(s, x-(t-s) y)|^{q}\right) \\
\leq & C\left(|\bar{t}-t|^{q}+|\bar{t}-t|^{q \kappa}\right) \leq C|\bar{t}-t|^{q \kappa} .
\end{aligned}
$$


Combining the estimates for $T_{3,1}, T_{3,2,1}$ and $T_{3,2,2}$ we conclude that

$$
T_{3} \leq C|\bar{t}-t|^{q \kappa} .
$$

Next we estimate the term $T_{4}$ which involves a stochastic integral. Consider the decomposition

$$
T_{4} \leq C\left(T_{4,1}+T_{4,2}\right)
$$

where

$$
T_{4,1}=\mathbb{E}\left|\int_{t}^{\bar{t}} \int_{\mathbb{R}^{3}} G(\bar{t}-s, x-d y) \sigma(s, y, u(s, y)) W(d s, d y)\right|^{q}
$$

and

$T_{4,2}=\mathbb{E}\left|\iint_{0}^{t}(G(\bar{t}-s, x-d y)-G(t-s, x-d y)) \sigma(s, y, u(s, y)) W(d s, d y)\right|^{q}$.

By the linear growth of $\sigma$ and Burkholder's inequality (Lemma 2.2), we obtain

$$
\begin{aligned}
T_{4,1} \leq C & \left(\int_{t}^{\bar{t}} d s \int_{\mathbb{R}^{3}} \int_{\mathbb{R}^{3}} G(\bar{t}-s, x-d y) G(\bar{t}-s, x-d z)\right. \\
& \times f(y-z) \sigma(s, y, u(s, y)) \sigma(s, z, u(s, z)))^{\frac{q}{2}} \\
=C & \left(\int_{0}^{\bar{t}-t} d s \int_{\mathbb{R}^{3}} G(s, x-d y) G(s, x-d z)\right. \\
& \times f(y-z) \sigma(\bar{t}-s, y, u(\bar{t}-s, y)) \sigma(\bar{t}-s, z, u(\bar{t}-s, z)))^{\frac{q}{2}} \\
\leq & \left(\int_{0}^{\bar{t}-t} d s \int_{\mathbb{R}^{3}} \int_{\mathbb{R}^{3}} G(s, x-d y) G(s, x-d z)\right. \\
& \times f(y-z)(1+|u(\bar{t}-s, y)|)(1+|u(\bar{t}-s, z)|))^{\frac{q}{2}} .
\end{aligned}
$$


Using Hölder's inequality, the moments estimate (2.15) and condition (1), we can write

$$
\begin{aligned}
T_{4,1} \leq & C(\bar{t}-t)^{\frac{q}{2}-1} \int_{0}^{\bar{t}-t} d s\left(\int_{\mathbb{R}^{3}} \int_{\mathbb{R}^{3}} G(s, x-d y) G(s, x-d z) f(y-z)\right)^{\frac{q}{2}} \\
& \times \sup _{s \in[0, T], y, z \in \mathbb{R}^{3}} \mathbb{E}\left((1+|u(s, y)|)^{\frac{q}{2}}(1+|u(s, z)|)^{\frac{q}{2}}\right) \\
\leq & C(\bar{t}-t)^{\frac{q}{2}-1} \int_{0}^{\bar{t}-t} d s\left(\int_{\mathbb{R}^{3}} G(s, d y) G(s, d z) f(y-z)\right)^{3} \\
\leq & C(\bar{t}-t)^{\frac{q}{2}-1} \int_{0}^{\bar{t}-t} d s\left(\int_{|z| \leq 2 s} \frac{f(z)}{|z|} d z\right)^{\frac{q}{2}} \\
\leq & C(\bar{t}-t)^{\frac{q}{2}-1} \int_{0}^{\bar{t}-t} s^{\nu \frac{q}{2}} d s=C(\bar{t}-t)^{q \frac{v+1}{2}} .
\end{aligned}
$$

For $T_{4,2}$, for notational convenience we denote $\bar{t}-t$ by $h$. Applying Burkholder's inequality (see Lemma 2.2) yields

$$
\begin{array}{rl}
T_{4,2} \leq C & \mathbb{E}\left(\int_{0}^{t} \int_{\mathbb{R}^{3}} \int_{\mathbb{R}^{3}}(G(h+s, d y)-G(s, d y))(G(h+s, d z)-G(s, d z))\right. \\
& \left.\times f(y-z) \Theta_{t, x}(s, y) \Theta_{t, x}(s, z) d s\right)^{\frac{q}{2}},
\end{array}
$$

where $\Theta_{t, x}(s, y)=\sigma(t-s, x-y, u(t-s, x-y))$. By making a change of variable, we can transform the integral in the space variable into an integral on the unit sphere $S^{2}$. In fact, denote $\xi=\frac{y}{|y|}$ and $\eta=\frac{z}{|z|}$ and we recall that $\sigma(d \xi)$ and $\sigma(d \eta)$ denote the uniform measure on $S^{2}$, so

$$
\begin{aligned}
& G(s, d y)=\frac{1}{4 \pi} s \sigma(d \xi), \\
& G(s, d z)=\frac{1}{4 \pi} s \sigma(d \eta) .
\end{aligned}
$$

After some rearrangements similar to those made for $Q$ in the proof of Theorem 3.2 (see also [5] for a similar strategy), we can write 


$$
T_{4,2}=C \mathbb{E}\left(R_{1}+R_{2}+R_{3}+R_{4}\right)^{\frac{q}{2}} \leq C \sum_{i=1}^{4} \mathbb{E}\left|R_{i}\right|^{\frac{q}{2}}
$$

where

$$
\begin{aligned}
R_{1}= & \int_{0}^{t} \int_{S^{2} \times S^{2}}(s+h)^{2} f((s+h) \xi-(s+h) \eta) \\
& \times\left(\Theta_{t, x}(s,(s+h) \xi)-\Theta_{t, x}(s, s \xi)\right)\left(\Theta_{t, x}(s,(s+h) \eta)\right. \\
& \left.-\Theta_{t, x}(s, s \eta)\right) \sigma(d \xi) \sigma(d \eta) d s, \\
R_{2}= & \int_{0}^{t} \int_{S^{2} \times S^{2}}\left((s+h)^{2} f((s+h) \xi-(s+h) \eta)-s(s+h) f(s \xi-(s+h) \eta)\right) \\
& \times\left(\Theta_{t, x}(s,(s+h) \eta)-\Theta_{t, x}(s, s \eta)\right) \Theta_{t, x}(s, s \xi) \sigma(d \xi) \sigma(d \eta) d s, \\
R_{3}= & \int_{0}^{t} \int_{S^{2} \times S^{2}}\left((s+h)^{2} f((s+h) \xi-(s+h) \eta)-s(s+h) f((s+h) \xi-s \eta)\right) \\
& \times\left(\Theta_{t, x}(s,(s+h) \xi)-\Theta_{t, x}(s, s \xi)\right) \Theta_{t, x}(s, s \eta) \sigma(d \xi) \sigma(d \eta) d s, \\
R_{4}= & \int_{0}^{t} \int_{S^{2} \times S^{2}}\left((s+h)^{2} f((s+h) \xi-(s+h) \eta)-s(s+h) f(s \xi-(s+h) \eta)\right. \\
& \left.-s_{s}(s+h) f((s+h) \xi-s \eta)+s^{2} f(s \xi-s \eta)\right) \Theta_{t, x}(s, s \xi) \\
& \times \Theta_{t, x}(s, s \eta) \sigma(d \xi) \sigma(d \eta) d s .
\end{aligned}
$$

We estimate each $\mathbb{E}\left|R_{i}\right|^{\frac{q}{2}}$ separately.

For $\mathbb{E}\left|R_{1}\right|^{\frac{q}{2}}$, using Hölder's inequality, the Lipschitz condition on $\sigma$, the assumption on the Hölder continuity on the space variable of $u$ (condition (2)), Lemma 6.4 and condition (2.5), we have

$$
\begin{aligned}
\mathbb{E}\left|R_{1}\right|^{\frac{q}{2}} & \leq C h^{q \kappa} \int_{0}^{t}\left(\int_{S^{2} \times S^{2}}(s+h)^{2} f((s+h) \xi-(s+h) \eta) \sigma(d \xi) \sigma(d \eta)\right)^{\frac{q}{2}} d s \\
& =C h^{q \kappa} \int_{0}^{t}\left(\int_{\mathbb{R}^{3}} \int_{\mathbb{R}^{3}} f(y-z) G(s+h, d y) G(s+h, d z)\right)^{\frac{q}{2}} d s \\
& =C h^{q \kappa} \int_{0}^{t}\left(\int_{|z| \leq 2(s+h)} \frac{f(z)}{|z|} d z\right)^{\frac{q}{2}} d s \\
& \leq C h^{q \kappa} .
\end{aligned}
$$


In order to estimate $\mathbb{E}\left|R_{2}\right|^{\frac{q}{2}}$, we make the decomposition

$$
\begin{array}{rl}
\mathbb{E}\left|R_{2}\right|^{\frac{q}{2}} \leq C & \mathbb{E}\left(\int_{0}^{t} \int_{S^{2} \times S^{2}} s(s+h)|f((s+h) \xi-(s+h) \eta)-f(s \xi-(s+h) \eta)|\right. \\
& \left.\times\left|\Theta_{t, x}(s,(s+h) \eta)-\Theta_{t, x}(s, s \eta)\right|\left|\Theta_{t, x}(s, s \xi)\right| \sigma(d \xi) \sigma(d \eta) d s\right)^{\frac{q}{2}} \\
& +C \mathbb{E}\left(\int_{0}^{t} \int_{S^{2} \times S^{2}} h(s+h) f((s+h) \xi-(s+h) \eta)\right. \\
& \left.\times\left|\Theta_{t, x}(s, s \xi)\right|\left|\Theta_{t, x}(s,(s+h) \eta)-\Theta_{t, x}(s, s \eta)\right| \sigma(d \xi) \sigma(d \eta) d s\right)^{\frac{q}{2}} \\
:=R_{2}^{1} & +R_{2}^{2} .
\end{array}
$$

For $R_{2}^{1}$, using the Hölder inequality, the Lipschitz and linear growth conditions on $\sigma$, the moments estimate (2.15), the assumption on the Hölder continuity in the space variable of $u$ (condition 2) and condition (4.1) with the change of variable $\eta \rightarrow-\eta$, we have

$$
\begin{aligned}
R_{2}^{1} \leq C & \left(\int_{0}^{t} \int_{S^{2} \times S^{2}} s(s+h) \mid f((s+h) \xi-(s+h) \eta)\right. \\
& -f(s \xi-(s+h) \eta) \mid \sigma(d \xi) \sigma(d \eta) d s)^{\frac{q}{2}} h^{\frac{q \kappa}{2}} \\
\leq C & \left(\int_{0}^{t} \int_{S^{2} \times S^{2}} s \mid f((s+h) \xi+(s+h) \eta)\right. \\
& -f(s \xi+(s+h) \eta) \mid \sigma(d \xi) \sigma(d \eta) d s)^{\frac{q}{2}} h^{\frac{q \kappa}{2}} \\
\leq C & h^{\frac{q \rho_{1}+q \kappa}{2}} .
\end{aligned}
$$

For $R_{2}^{2}$, using Hölder's inequality, the Lipschitz condition and linear growth conditions on $\sigma$, the moments estimate (2.15), the assumption on the Hölder continuity in the space variable (condition (2)) and condition (1), we have 


$$
\begin{aligned}
R_{2}^{2} & \leq C\left(\int_{0}^{t} \int_{S^{2} \times S^{2}}(s+h) f((s+h) \xi-(s+h) \eta) \sigma(d \xi) \sigma(d \eta) d s\right)^{\frac{q}{2}} h^{\frac{q+q \kappa}{2}} \\
& \leq C\left(\int_{0}^{t} \frac{1}{s+h} \int_{\mathbb{R}^{3}} \int_{\mathbb{R}^{3}} f(y-z) G(s+h, d y) G(s+h, d z) d s\right)^{\frac{q}{2}} h^{\frac{q+q \kappa}{2}} \\
& =C\left(\int_{0}^{t} \frac{1}{s+h} \int_{|z| \leq 2(s+h)} \frac{f(z)}{|z|} d z d s\right)^{\frac{q}{2}} h^{\frac{q+q \kappa}{2}} \\
& \leq C h^{\frac{q+q \kappa}{2}} .
\end{aligned}
$$

Combining the estimates for $R_{2}^{1}$ and $R_{2}^{2}$, we have

$$
\mathbb{E}\left|R_{2}\right|^{\frac{q}{2}} \leq C h^{\frac{q\left(\rho_{1}+\kappa\right)}{2}} .
$$

Similarly,

$$
\mathbb{E}\left|R_{3}\right|^{\frac{q}{2}} \leq C h^{\frac{q\left(\rho_{1}+\kappa\right)}{2}} .
$$

For $R_{4}$, using the linear growth of $\sigma$, the moments estimate (2.15) and the change of variable $\eta \rightarrow-\eta$, we have

$$
\begin{aligned}
\mathbb{E}\left|R_{4}\right|^{\frac{q}{2}} \leq C & \left(\int_{0}^{t} \int_{S^{2} \times S^{2}} \mid(s+h)^{2} f((s+h) \xi-(s+h) \eta)-s(s+h) f(s \xi-(s+h) \eta)\right. \\
& \left.-s(s+h) f((s+h) \xi-s \eta)+s^{2} f(s \xi-s \eta) \mid \sigma(d \xi) \sigma(d \eta) d s\right)^{\frac{q}{2}} \\
\leq C & \left(\int_{0}^{t} \int_{S^{2} \times S^{2}} s^{2} \mid f((s+h) \xi+(s+h) \eta)-f(s \xi+(s+h) \eta)\right. \\
& -f((s+h) \xi+s \eta)+f(s \xi+s \eta) \mid \sigma(d \xi) \sigma(d \eta) d s)^{\frac{q}{2}} \\
& +C\left(\int_{0}^{t} \int_{S^{2} \times S^{2}}^{t} s h \mid f((s+h) \xi+(s+h) \eta)\right.
\end{aligned}
$$




$$
\begin{aligned}
& -f(s \xi+(s+h) \eta) \mid \sigma(d \xi) \sigma(d \eta) d s)^{\frac{q}{2}} \\
& +C\left(\int_{0}^{t} \int_{S^{2} \times S^{2}} s h \mid f((s+h) \xi+(s+h) \eta)\right. \\
& -f((s+h) \xi+s \eta) \mid \sigma(d \xi) \sigma(d \eta) d s)^{\frac{q}{2}} \\
& +C\left(\int_{0}^{t} \int_{S^{2} \times S^{2}} h^{2} f((s+h) \xi-(s+h) \eta) \sigma(d \xi) \sigma(d \eta) d s\right)^{\frac{q}{2}} \\
& :=R_{4}^{1}+R_{4}^{2}+R_{4}^{3}+R_{4}^{4} .
\end{aligned}
$$

For $R_{4}^{1}$, condition (4.2) yields

$$
R_{4}^{1} \leq C h^{\frac{q \rho_{2}}{2}} .
$$

For $R_{4}^{2}$ and $R_{4}^{3}$, applying condition (4.1) we obtain

$$
R_{4}^{2} \leq C h^{\frac{q \rho_{1}+q}{2}}, \quad R_{4}^{3} \leq C h^{\frac{q \rho_{1}+q}{2}} .
$$

For $R_{4}^{4}$, condition (1) allows us to write

$$
\begin{aligned}
R_{4}^{4} & =C h^{q}\left(\int_{0}^{t} \int_{|z| \leq 2(s+h)} \frac{1}{(s+h)^{2}} \frac{f(z)}{|z|} d z d s\right)^{\frac{q}{2}} \\
& \leq C h^{q}\left(\int_{0}^{t}(s+h)^{-2+v} d s\right)^{\frac{q}{2}} .
\end{aligned}
$$

When $v<1, R_{4}^{4} \leq C h^{\frac{q(v+1)}{2}}$, when $v=1, R_{4}^{4} \leq C h^{q}(\log (t+h)-\log h)^{\frac{q}{2}} \leq$ $C h^{q}(\log (T+h)-\log h)^{\frac{q}{2}} \leq C h^{q(1-\varepsilon)}$ for any $\varepsilon>0$.

Combining the estimates for $R_{4}^{1}, R_{4}^{2}, R_{4}^{3}, R_{4}^{4}$, we have

$$
\mathbb{E}\left|R_{4}\right|^{\frac{q}{2}} \leq C\left(h^{\frac{q \rho_{2}}{2}}+h^{\frac{q \rho_{1}+q}{2}}+h^{q \frac{\nu+1}{2}}+h^{q(1-\varepsilon)}\right),
$$

for any $\varepsilon>0$. By (4.5), (4.6), (4.7), (4.8) and (4.9), we conclude that

$$
T_{4} \leq C h^{q \rho}
$$


where $0<\rho<\min \left(\frac{\nu+1}{2}, \frac{\rho_{1}+\kappa}{2}, \frac{\rho_{2}}{2}, \kappa\right)$. From the proof it is easy to see that the constant $C$ in the above expression does not depend on $x$. Then we combine the estimates of (4.3), (4.4) and (4.10) to obtain

$$
\sup _{x \in \mathbb{R}^{3}} \mathbb{E}|u(t, x)-u(\bar{t}, x)|^{q} \leq C|\bar{t}-t|^{q \kappa^{\prime}},
$$

where $\kappa^{\prime} \in\left(0, \min \left(\gamma_{1}, \gamma_{2}, \frac{v+1}{2}, \frac{\rho_{1}+\kappa}{2}, \frac{\rho_{2}}{2}, \kappa\right)\right)$.

An application of Kolmogorov's continuity criteria leads to the following Hölder continuity result in the space an time variables.

Corollary 4.2 Let $u$ be the solution to Eq. (1.1). Assume conditions (a) and (b) in Theorem 3.1. Suppose that condition (c) of Theorem 3.1 or condition (a) of Theorem 3.2 hold. Set $\kappa_{1}=\min \left(\gamma_{1}, \gamma_{2}, \gamma, \frac{\gamma^{\prime}}{2}\right)$ in the first case and $\kappa_{1}=\min \left(\gamma_{1}, \gamma_{2}, \gamma\right)$ in the second case. Suppose also that conditions (1), (2) and (3) of Theorem 3.2 hold. Set $\kappa_{2}=\min \left(\gamma_{1}, \gamma_{2}, \kappa_{1}, \frac{v+1}{2}, \frac{\rho_{1}+\kappa_{1}}{2}, \frac{\rho_{2}}{2}\right)$. Then, for any $\kappa<\kappa_{1}$ and $\kappa^{\prime}<\kappa_{2}$ there exists a version of the process $u$ which is locally Hölder continuous of order $\kappa$ in the space variable and of order $\kappa^{\prime}$ in the time variable. That is, for any bounded rectangle $I \subset \mathbb{R}^{3}$ we can find a random variable $K_{\kappa, \kappa^{\prime}, I}$ such that

$$
|u(t, x)-u(s, y)| \leq K_{\kappa, \kappa^{\prime}, I}\left(|t-s|^{\kappa^{\prime}}+|x-y|^{\kappa}\right)
$$

for all $s, t \in[0, T]$ and $x, y \in I$.

\section{Examples}

In this section, we give some examples of covariance functions $f$ satisfying the conditions in the previous theorems.

\subsection{Example 1}

Proposition 5.1 Let $f$ be a non-negative and non-negative definite $C^{2}$ function. Then condition (c) of Theorem 3.1 holds with $\gamma=1$ and $\gamma^{\prime}=2$.

Proof Using some basic estimate from calculus, we have

$$
\int_{|z| \leq 2 T} \frac{|f(z+w)-f(z)|}{|z|} d z \leq C \int_{|z| \leq 2 T+1} \frac{\sup _{|z| \leq 2 T+1}|\nabla f(z)|}{|z|}|w| d z \leq C|w|,
$$

and

$$
\int_{|z| \leq 2 T} \frac{|f(z+w)+f(z-w)-2 f(z)|}{|z|} d z
$$




$$
\leq C \int_{|z| \leq 2 T+1} \max _{1 \leq i \leq 3,1 \leq j \leq 3} \sup _{|z| \leq 2 T+1}\left|\frac{\partial^{2} f}{\partial z_{i} \partial z_{j}}\right| \frac{1}{|z|}|w|^{2} d z \leq C|w|^{2} .
$$

The claim follows.

Remark 5.2 Consider the example $f(x)=\left(\rho * \frac{1}{|\cdot|^{\beta}}\right)(x)$, where $\rho(x)$ is a nonnegative Schwartz function defined in $\mathbb{R}^{3}$ such that $\left(\mathcal{F}^{-1} \rho\right)(\xi) \geq 0$ (for example, $\rho(x)=$ $e^{-|x|^{2}}$ ) and $0<\beta<3$. Then it is easy to see that condition $\left(c^{\prime}\right)$ of Theorem 3.2 holds for $0<\gamma<\min \left(\frac{3-\beta}{2}, 1\right)$. The restriction $\gamma<\frac{3-\beta}{2}$ comes from the fact that under the condition $0<\beta<3$, the Fourier transform of $\frac{1}{|x|^{\beta}}$ is $\frac{C_{\beta}}{|\xi|^{3-\beta}}$ for some constant $C_{\beta}$ which only depends on $\beta$. We omit the details of the proof. Notice in this example, Theorem 3.2 gives a weaker result than what we would obtain using Theorem 3.1 as we have done in Proposition 5.1.

\subsection{The Riesz kernel}

Before giving next example, we recall some results from Dalang and Sanz-Solé [5].

Let $\xi, \eta$ be two unit vectors in $\mathbb{R}^{3}$ and let $u$ be any point in $\mathbb{R}^{3}$. Suppose $a, b$ are positive numbers with $a+b \in(0,3)$. Then we have for any $h \in \mathbb{R}$

$$
|u+h \xi|^{a+b-3}-|u|^{a+b-3}=|h|^{b} \int_{\mathbb{R}^{3}} d w|u-h w|^{a-3}\left(|w+\xi|^{b-3}-|w|^{b-3}\right),
$$

and

$$
\begin{aligned}
& || u+h \xi+\left.h \eta\right|^{a+b-3}-|u+h \xi|^{a+b-3}-|u+h \eta|^{a+b-3}+|u|^{a+b-3} \mid \\
& \leq|h|^{b} \int_{\mathbb{R}^{d}} d w|u-h w|^{a-3}|| w+h \xi+\left.h \eta\right|^{b-3}-|w+h \xi|^{b-3} \\
& \quad-|w+h \eta|^{b-3}+|w|^{b-3} \mid .
\end{aligned}
$$

Proposition 5.3 Let $f(x)=|x|^{-\beta}, 0<\beta<2$. Then $f$ satisfies condition $\left(c^{\prime}\right)$ in Theorem 3.2 for any $\gamma \in\left(0, \frac{2-\beta}{2}\right)$ and $f$ also satisfies conditions (1), (4.1) and (4.2) in Theorem 4.1 for $v=2-\beta$, any $0<\rho_{1}<\min \left(2-\beta\right.$, 1) and $0<\rho_{2}<2-\beta$.

Proof Let us first check condition $\left(c^{\prime}\right)$ in Theorem 3.2. Since $f(x)=|x|^{-\beta}$, we have $\mu(d \xi)=C|\xi|^{-3+\beta} d \xi$. Then it is easy to see that

$$
\int_{\mathbb{R}^{3}} \frac{\mu(d \xi)}{1+|\xi|^{2-2 \gamma}}<\infty
$$


since $0<\gamma<\frac{2-\beta}{2}$, and we have

$$
\mathcal{F}\left(|\xi|^{2 \gamma} \mu(d \xi)\right)(x)=C \mathcal{F}\left(|\xi|^{-3+\beta+2 \gamma} d \xi\right)(x)=C|x|-(\beta+2 \gamma)
$$

for some positive constant $C$, so the above expression is nonnegative. So, condition $\left(c^{\prime}\right)$ in Theorem 3.2 holds.

To verify condition (1) in Theorem 4.1, we notice

$$
\int_{|z| \leq h} \frac{f(z)}{|z|} d z=\int_{|z| \leq h}|z|^{-\beta-1} d z=C h^{2-\beta} .
$$

So condition (1) in Theorem 4.1 is satisfied with $v=2-\beta$.

We turn to condition (4.1). We apply (5.1) with $b=\rho_{1}<\min ((2-\beta), 1), d=3$, $a=3-\rho_{1}-\beta, u=s(\xi+\eta)+h \eta$ to get

$$
\begin{aligned}
& \int_{0}^{T} \int_{S^{2}} \int_{S^{2}} s|f(s(\xi+\eta)+h(\xi+\eta))-f(s(\xi+\eta)+h \eta)| \sigma(d \xi) \sigma(d \eta) d s \\
& \leq h^{\rho_{1}} \int_{0}^{T} \int_{S^{2} \times S^{2}} s \int_{\mathbb{R}^{3}} d w|s \xi+(s+h) \eta-h w|^{-\rho_{1}-\beta}|| w+\left.\xi\right|^{\rho_{1}-3} \\
& -|w|^{\rho_{1}-3} \mid \sigma(d \xi) \sigma(d \eta) d s \\
& \leq h^{\rho_{1}} \int_{0}^{T} \int_{S^{2} \times S^{2}} s \int_{|w| \leq 3} d w|s \xi+(s+h) \eta-h w|^{-\rho_{1}-\beta}|w+\xi|^{\rho_{1}-3} \sigma(d \xi) \sigma(d \eta) d s \\
& +h^{\rho_{1}} \int_{0}^{T} \int_{S^{2} \times S^{2}} s \int_{|w| \leq 3} d w|s \xi+(s+h) \eta-h w|^{-\rho_{1}-\beta}|w|^{\rho_{1}-3} \sigma(d \xi) \sigma(d \eta) d s \\
& +h^{\rho_{1}} \int_{0}^{T} \int_{S^{2} \times S^{2}} s \int_{|w|>3} d w|s \xi+(s+h) \eta-h w|^{-\rho_{1}-\beta} \\
& +\left.|| w \xi\right|^{\rho_{1}-3}-|w|^{\rho_{1}-3} \mid \sigma(d \xi) \sigma(d \eta) d s \\
& :=h^{\rho_{1}}\left(I_{1}+I_{2}+I_{3}\right) \text {. }
\end{aligned}
$$

For $I_{1}$, making the change of variable $w+h \rightarrow w$, using the Fourier transform (see Lemma 6.5) and noting that $I_{1}$ is real positive, we can write: 


$$
\begin{aligned}
I_{1} & \leq \int_{0}^{T} \int_{S^{2} \times S^{2}} s \int_{|w| \leq 4}|(s+h) \xi+(s+h) \eta-h w|^{-\rho_{1}-\beta}|w|^{\rho_{1}-3} d w \sigma(d \xi) \sigma(d \eta) d s \\
& =C \int_{0}^{T} \int_{|w| \leq 4} \int_{\mathbb{R}^{3} \times \mathbb{R}^{3}} \frac{s}{(s+h)^{2}}|y+z-h w|^{-\rho_{1}-\beta} G(s+h, d y) G(s+h, d z)|w|^{\rho_{1}-3} d w d s \\
& =C \int_{0}^{T} \int_{\mathbb{R}^{3}} \frac{s}{(s+h)^{2}} \frac{[\sin ((s+h)|\xi|)]^{2}}{|\xi|^{2}}|\xi|^{-3+\rho_{1}+\beta} e^{i \xi \cdot h w} d \xi d s \int_{|w| \leq 4}|w|^{\rho_{1}-3} d w .
\end{aligned}
$$

Then using the change of variable $(s+h) \xi=\eta$ and the bound $\left|e^{i \xi \cdot h w}\right| \leq 1$, by direct calculation we see that $I_{1}<\infty$.

For $I_{2}$, we do the same calculation, but we do not need the change of variable for $w$. Let $2 \varepsilon<2-\beta-\rho_{1}$, then

$$
\begin{aligned}
I_{2} \leq & \int_{0}^{T} \int_{\mathbb{R}^{3}} \frac{1}{s+h} \frac{|\sin ((s+h)|\xi|) \sin (s|\xi|)|}{|\xi|^{2}}|\xi|^{-3+\rho_{1}+\beta} d \xi d s \int_{|w| \leq 3}|w|^{\rho_{1}-3} d w \\
\leq & C \int_{0}^{T} \int_{|\xi| \leq 1} s|\xi|^{-3+\rho_{1}+\beta} d \xi d s \\
& +C \int_{0}^{T} \int_{|\xi|>1} \frac{1}{s+h} \frac{(s+h)^{\varepsilon} s^{\varepsilon}|\xi|^{2 \varepsilon}}{|\xi|^{2}}|\xi|^{-3+\rho_{1}+\beta} d \xi d s
\end{aligned}
$$

which is finite by direct calculation.

For $I_{3}$ we can write

$$
\begin{aligned}
I_{3}= & \int_{0} \int_{|w|>3} \int_{S^{2} \times S^{2}} s \mid s \xi+(s+h) \eta \\
& -\left.h w\right|^{-\rho_{1}-\beta} \sigma(d \xi) \sigma(d \eta) d w\left|\int_{0}^{1} \frac{d}{d \lambda}\right| w+\left.\lambda \xi\right|^{\rho_{1}-3} d \lambda \mid d s \\
\leq & \int_{0}^{T} \int_{|w|>3} \int_{S^{2} \times S^{2}} s \mid s \xi+(s+h) \eta \\
& -\left.h w\right|^{-\rho_{1}-\beta} \sigma(d \xi) \sigma(d \eta)\left(\int_{0}^{1}|w|^{\rho_{1}-4} d \lambda\right) d w d s,
\end{aligned}
$$


where the inequality holds because $|w|>3,0 \leq \lambda \leq 1$ and $|\xi|=1$. We can show that $I_{3}<\infty$ similarly to the proof for $I_{2}$ using the fact that $\int_{|w|>3}|w|^{\rho_{1}-4} d w<\infty$ since $\rho_{1}<1$.

It is easy to see that $I_{1}, I_{2}$ and $I_{3}$ are finite uniformly for $0<h \leq 1$. Therefore, condition (4.1) is satisfied with $0<\rho_{1}<\min (2-\beta, 1)$.

For condition (4.2), applying (5.2), with $d=3, b=\rho_{2}<2-\beta, a=3-\rho_{2}-\beta$, $u=s(\xi+\eta)$, yields

$$
\begin{aligned}
& \int_{0}^{T} \int_{S^{2}} \int_{S^{2}} \mid f(s(\xi+\eta)+h(\xi+\eta))-f(s(\xi+\eta)+h \xi) \\
& -f(s(\xi+\eta)+h \eta)+f(s(\xi+\eta)) \mid \times s^{2} \sigma(d \xi) \sigma(d \eta) \\
& \leq h^{\rho_{2}} \int_{0}^{T} \int_{S^{2} \times S^{2}} s^{2} \int_{\mathbb{R}^{3}}|s(\xi+\eta)-h w|^{-\rho_{2}-\beta} \\
& \times|| w+\xi+\left.\eta\right|^{\rho_{2}-3}-|w+\xi|^{\rho_{2}-3}-|w+\eta|^{\rho_{2}-3}+|w|^{\rho_{2}-3} \mid d w \sigma(d \xi) \sigma(d \eta) d s \\
& \leq h^{\rho_{2}}\left(\int_{0}^{T} \int_{S^{2} \times S^{2}} s^{2} \int_{|w| \leq 3}|s(\xi+\eta)-h w|^{-\rho_{2}-\beta}|w+\xi+\eta|^{\rho_{2}-3} d w \sigma(d \xi) \sigma(d \eta) d s\right. \\
& +\int_{0}^{T} \int_{S^{2} \times S^{2}} s^{2} \int_{|w| \leq 3}|s(\xi+\eta)-h w|^{-\rho_{2}-\beta}|w+\xi|^{\rho_{2}-3} d w \sigma(d \xi) \sigma(d \eta) d s \\
& +\int_{0}^{T} \int_{S^{2} \times S^{2}} s^{2} \int_{|w| \leq 3}|s(\xi+\eta)-h w|^{-\rho_{2}-\beta}|w+\eta|^{\rho_{2}-3} d w \sigma(d \xi) \sigma(d \eta) d s \\
& +\int_{0}^{T} \int_{S^{2} \times S^{2}} s^{2} \int_{|w| \leq 3}|s(\xi+\eta)-h w|^{-\rho_{2}-\beta}|w|^{\rho_{2}-3} d w \sigma(d \xi) \sigma(d \eta) d s \\
& +\int_{0}^{T} \int_{S^{2} \times S^{2}} s^{2} \int_{|w|>3}|s(\xi+\eta)-h w|^{-\rho_{2}-\beta} \\
& \left.\times|| w+\xi+\left.\eta\right|^{\rho_{2}-3}-|w+\xi|^{\rho_{2}-3}-|w+\eta|^{\rho_{2}-3}+|w|^{\rho_{2}-3} \mid d w \sigma(d \xi) \sigma(d \eta) d s\right) \\
& :=h^{\rho_{2}}\left(\sum_{i=1}^{5} L_{i}\right)
\end{aligned}
$$

For $L_{i}, i=1,2,3,4$, we can proceed exactly in the same way as for the integrals $I_{1}, I_{2}$ above. For $L_{5}$, we can express 


$$
\begin{aligned}
\mid w & +\xi+\left.\eta\right|^{\rho_{2}-3}-|w+\xi|^{\rho_{2}-3}-|w+\eta|^{\rho_{2}-3}+|w|^{\rho_{2}-3} \\
= & \int_{0}^{1} \int_{0}^{1} \frac{\partial^{2}}{\partial \lambda \partial \mu}|w+\lambda \xi+\mu \eta|^{\rho_{2}-3} d \lambda d \mu,
\end{aligned}
$$

and since $|w|>3,|\eta|=1$, it is easy to see that

$$
\left|\frac{\partial^{2}}{\partial \lambda \partial \mu}\right| w+\lambda \xi+\left.\left.\mu \eta\right|^{\rho_{2}-3}|\leq C| w\right|^{\rho_{2}-5} .
$$

So $\int_{|w|>3}|w|^{\rho_{2}-5} d w$ is finite, and $L_{5}$ is finite, by the same argument as for $I_{3}$.

So condition (4.2) is satisfied with $0<\rho_{2}<2-\beta$. This completes the proof.

Notice that, with the notation of Corollary 4.2, for the Riesz kernel we can take $\kappa_{1}=\kappa_{2}<\frac{2-\beta}{2}$, and we deduce the local Hölder continuity of the solution $u$ in space and time variables of order $\kappa<\min \left(\gamma_{1}, \gamma_{2}, \frac{2-\beta}{2}\right)$. In this way we recover the result by Dalang and Sanz-Solé [5].

\subsection{The Bessel kernel}

In this subsection we consider the Bessel kernel defined by

$$
f(x)=\int_{0}^{\infty} w^{\frac{\alpha-5}{2}} e^{-w} e^{-\frac{|x|^{2}}{4 w}} d w
$$

for some $\alpha>1$. The colored noise with this covariance has received some attention in the literature (see for example, $[1,2]$ ).

Proposition 5.4 Let $f$ be given by (5.3). Then $f$ satisfies (2.5), (3.1), (3.2), (4.1), (4.2) and condition (1) in Theorem 4.1 for any $0<\gamma, \rho_{1}, v<\min (\alpha-1,1)$ and $0<\gamma^{\prime}, \rho_{2}<\min (\alpha-1,2)$.

Proof First let us check condition (2.5). We have

$$
\int_{|x| \leq 1} \frac{f(x)}{|x|} d x=\int_{0}^{\infty} w^{\frac{\alpha-5}{2}} e^{-w} \int_{|x| \leq 1} \frac{e^{-\frac{|x|^{2}}{4 w}}}{|x|} d x d w .
$$

The change of variable $x=2 \sqrt{w} y$ gives

$$
\int_{|x| \leq 1} \frac{f(x)}{|x|} d x=4 \int_{0}^{\infty} w^{\frac{\alpha-3}{2}} e^{-w} \int_{|y| \leq \frac{1}{2 \sqrt{w}}} \frac{e^{-|y|^{2}}}{|y|} d y \leq C \int_{0}^{\infty} w^{\frac{\alpha-3}{2}} e^{-w} d w<\infty
$$


because $\alpha>1$. To check condition (3.1), we note that for $a, b \geq 0$, we have $\left|e^{-a}-e^{-b}\right| \leq|a-b|^{\gamma}\left(e^{-a} \vee e^{-b}\right)$, for any $0 \leq \gamma \leq 1$. So

$$
\begin{aligned}
\left|e^{-\frac{|z+y|^{2}}{4 w}}-e^{-\frac{|z|^{2}}{4 w}}\right| & \leq\left(\frac{1}{4 w}\right)^{\gamma}|| z+\left.y\right|^{2}-\left.|z|^{2}\right|^{\gamma}\left(e^{-\frac{|z+y|^{2}}{4 w}} \vee e^{-\frac{|z|^{2}}{4 w}}\right) \\
& \leq C|y|^{\gamma}\left(|z+y|^{\gamma}+|z|^{\gamma}\right) \frac{1}{w^{\gamma}}\left(e^{-\frac{|z+y|^{2}}{4 w}}+e^{-\frac{|z|^{2}}{4 w}}\right) .
\end{aligned}
$$

As a consequence

$$
\begin{aligned}
& \int_{|z| \leq 2 T} \frac{|f(z+y)-f(z)|}{|z|} d z \\
& \leq|y|^{\gamma} \int_{0}^{\infty} w^{\frac{\alpha-5}{2}-\gamma} e^{-w}\left(\int_{|z| \leq 2 T}\left(|z+y|^{\gamma}+|z|^{\gamma}\right) \frac{e^{-\frac{|z+y|^{2}}{4 w}}}{|z|} d z\right. \\
& \left.\quad+\int_{|z| \leq 2 T}\left(|z+y|^{\gamma}+|z|^{\gamma}\right) \frac{e^{-\frac{|z|^{2}}{4 w}}}{|z|} d z\right) d w \\
& :=|y|^{\gamma} \int_{0}^{\infty} w^{\frac{\alpha-5}{2}-\gamma} e^{-w}(I(y)+J(y)) d w .
\end{aligned}
$$

For the integral $I(y)$, with the change of variable $z=\sqrt{w} x-y$, we have

$$
\begin{aligned}
I(y) & =\int_{\left|x-\frac{y}{\sqrt{w}}\right| \leq \frac{2 T}{\sqrt{w}}} w^{\frac{\gamma+2}{2}}\left(\frac{|x|^{\gamma}}{\left|x-\frac{y}{\sqrt{w}}\right|}+\left|x-\frac{y}{\sqrt{w}}\right|^{\gamma-1}\right) e^{-\frac{|x|^{2}}{4}} d x \\
& \leq \int_{\mathbb{R}^{3}} w^{\frac{\gamma+2}{2}}\left(\frac{|x|^{\gamma}}{\left|x-\frac{y}{\sqrt{w}}\right|}+\left|x-\frac{y}{\sqrt{w}}\right|^{\gamma-1}\right) e^{-\frac{|x|^{2}}{4}} d x \\
& \leq C w^{\frac{\gamma+2}{2}}
\end{aligned}
$$

where the last inequality follows from the fact that $|x|^{\gamma} e^{-\frac{|x|^{2}}{4}} \leq C e^{-\frac{|x|^{2}}{8}}$ and Lemma 17 in [6]. The term $J(y)$ can be estimated in the same way using the change of variable $z=\sqrt{w} y$, and we have

$$
J(y) \leq C w^{\frac{\gamma+2}{2}} .
$$


Hence,

$$
\int_{|z| \leq 2 T} \frac{|f(z+y)-f(z)|}{|z|} d z \leq C|y|^{\gamma} \int_{0}^{\infty} w^{\frac{\alpha-\gamma-3}{2}} e^{-w} d w \leq C|y|^{\gamma}
$$

for any $0<\gamma<\alpha-1$. So condition (3.1) is satisfied with $0<\gamma<\min (\alpha-1,1)$.

To check condition (3.2), note that

$$
|f(z+y)+f(z-y)-2 f(z)|=\left|\int_{0}^{1} \int_{0}^{1} \frac{\partial^{2}}{\partial \lambda \partial \mu} f(z-(\lambda-\mu) y) d \lambda d \mu\right| .
$$

So we have

$$
\begin{aligned}
& \left|e^{-\frac{|z+y|^{2}}{4 w}}+e^{-\frac{|z-y|^{2}}{4 w}}-2 e^{-\frac{|z|^{2}}{4 w}}\right| \\
\leq & \int_{0}^{1} \int_{0}^{1}\left(e^{-\frac{|z-\lambda y+\mu y|^{2}}{4 w}} \frac{1}{4 w^{2}}((z-\lambda y+\mu y) \cdot y)^{2}+e^{-\frac{|z-\lambda y+\mu y|^{2}}{4 w}} \frac{1}{2 w}|y|^{2}\right) d \lambda d \mu \\
\leq & \int_{0}^{1} \int_{0}^{1} e^{-\frac{|z-\lambda y+\mu y|^{2}}{4 w}}\left(\frac{1}{4 w^{2}}|z-\lambda y+\mu y|^{2}|y|^{2}+\frac{1}{2 w}|y|^{2}\right) d \lambda d \mu \\
\leq & C \int_{0}^{1} 1 \int_{0}^{1} \frac{|y|^{2}}{w} e^{-\frac{|z-(\lambda-\mu) y|^{2}}{8 w}} d \lambda d \mu .
\end{aligned}
$$

Here we have used the fact that $x^{2} e^{-x^{2}} \leq C e^{-\frac{x^{2}}{2}}$. By considering the cases $\frac{|y|}{\sqrt{w}} \leq 1$ and $\frac{|y|}{\sqrt{w}}>1$, we obtain

$$
\begin{aligned}
& \left|e^{-\frac{|z+y|^{2}}{4 w}}+e^{-\frac{|z-y|^{2}}{4 w}}-2 e^{-\frac{|z|^{2}}{4 w}}\right| \\
& \leq C \frac{|y|^{\gamma^{\prime}}}{w^{\gamma^{\prime} / 2}} \int_{0}^{1} \int_{0}^{1}\left(e^{-\frac{|z-(\lambda-\mu) y|^{2}}{8 w}}+e^{-\frac{|z+y|^{2}}{4 w}}+e^{-\frac{|z-y|^{2}}{4 w}}+2 e^{-\frac{|z|^{2}}{4 w}}\right) d \lambda d \mu
\end{aligned}
$$

for any $0 \leq \gamma^{\prime} \leq 2$. So we have

$$
\int_{|z| \leq 2 T} \frac{|f(z+y)+f(z-y)-2 f(z)|}{|z|} d z
$$




$$
\begin{aligned}
& \leq C|y|^{\gamma^{\prime}} \int_{|z| \leq 2 T} \int_{0}^{\infty} d w \int_{0}^{1} d \lambda \int_{0}^{1} d \mu w^{\frac{\alpha-5-\gamma^{\prime}}{2}} e^{-w} \\
& \quad \times\left(e^{-\frac{|z-(\lambda-\mu) y|^{2}}{8 w}}+e^{-\frac{|z+y|^{2}}{4 w}}+e^{-\frac{|z-y|^{2}}{4 w}}+2 e^{-\frac{|z|^{2}}{4 w}}\right) \frac{1}{|z|} d z .
\end{aligned}
$$

By Lemma 17 in [6], we can write

$$
\int_{|z| \leq 2 T}\left(e^{-\frac{|z-(\lambda-\mu) y|^{2}}{8 w}}+e^{-\frac{|z+y|^{2}}{4 w}}+e^{-\frac{|z-y|^{2}}{4 w}}+2 e^{-\frac{|z|^{2}}{4 w}}\right) \frac{1}{|z|} d z \leq C w,
$$

where the constant $C$ does not depend on $y, \lambda, \mu$. Therefore,

$$
\int_{|z| \leq 2 T} \frac{|f(z+y)+f(z-y)-2 f(z)|}{|z|} d z \leq C|y|^{\gamma^{\prime}} \int_{0}^{\infty} w^{\frac{\alpha-3-\gamma^{\prime}}{2}} e^{-w} d w \leq C|y|^{\gamma^{\prime}}
$$

for any $0<\gamma^{\prime}<\min (\alpha-1,2)$. As a consequence, condition (3.2) is satisfied with $0<\gamma^{\prime}<\min (\alpha-1,2)$.

To check condition (1) in Theorem 4.1 holds we compute

$$
\int_{|x| \leq h} \frac{e^{-\frac{|x|^{2}}{4 w}}}{|x|} d x=4 \pi \int_{0}^{h} e^{-\frac{r^{2}}{4 w}} r d r=8 \pi w\left(1-e^{-\frac{h^{2}}{4 w}}\right) \leq C h^{\nu} w^{1-\frac{\nu}{2}}
$$

for any $0 \leq v \leq 1$. This implies that

$$
\int_{|x| \leq h} \frac{f(x)}{|x|} d x \leq C h^{v} \int_{0}^{\infty} w^{\frac{\alpha-3-v}{2}} e^{-w} d w \leq C h^{v}
$$

for any $0<v<\min (\alpha-1,1)$. So condition (1) in Theorem 4.1 is satisfied with $0<v<\min (\alpha-1,1)$.

To check the condition (4.1), first we note that

$$
\begin{aligned}
& \left|e^{-\frac{|x+h \xi|^{2}}{4 w}}-e^{-\frac{|x|^{2}}{4 w}}\right|=\left|\int_{0}^{1} \frac{d}{d \lambda} e^{-\frac{|x+\lambda h \xi|^{2}}{4 w}} d \lambda\right|=\left|\int_{0}^{1} e^{-\frac{|x+\lambda h \xi|^{2}}{4 w}} \frac{\langle x+\lambda h \xi, h \xi\rangle}{2 w} d \lambda\right| \\
\leq & C \int_{0}^{1} e^{-\frac{|x+\lambda h \xi|^{2}}{4 w}} \frac{|x+\lambda h \xi|}{\sqrt{w}} \frac{h}{\sqrt{w}} d \lambda \leq C \int_{0}^{1} e^{-\frac{|x+\lambda h \xi|^{2}}{8 w}} \frac{h}{\sqrt{w}} d \lambda,
\end{aligned}
$$


where we have used the fact that $|x| e^{-x^{2}} \leq C e^{-\frac{x^{2}}{2}}$. By considering the cases $\frac{h}{\sqrt{w}} \leq 1$ and $\frac{h}{\sqrt{w}}>1$, we can write

$$
\left|e^{-\frac{|x+h \xi|^{2}}{4 w}}-e^{-\frac{|x|^{2}}{4 w}}\right| \leq C\left(\frac{h}{\sqrt{w}}\right)^{\rho_{1}} \int_{0}^{1}\left(e^{-\frac{|x+\lambda h \xi|^{2}}{8 w}}+e^{-\frac{|x+h \xi|^{2}}{4 w}}+e^{-\frac{|x|^{2}}{4 w}}\right) d \lambda
$$

for any $\rho_{1} \in[0,1]$. So we have

$$
|f(x+h \xi)-f(x)| \leq C h^{\rho_{1}} \int_{0}^{1} \int_{0}^{\infty} w^{\frac{\alpha-5-\rho_{1}}{2}} e^{-w}\left(e^{-\frac{|x+\lambda h \xi|^{2}}{8 w}}+e^{-\frac{|x+h \xi|^{2}}{4 w}}+e^{-\frac{|x|^{2}}{4 w}}\right) d w d \lambda .
$$

Therefore, for any $\rho_{1} \in[0,1]$.

$$
\begin{aligned}
& \int_{0}^{T} \int_{S^{2}} \int_{S^{2}}|f(s(\xi+\eta)+h(\xi+\eta))-f(s(\xi+\eta)+h \eta)| s \sigma(d \xi) \sigma(d \eta) d s \\
& \leq C h^{\rho_{1}} \int_{0}^{1} \int_{0}^{\infty} w^{\frac{\alpha-5-\rho_{1}}{2}} e^{-w} \int_{0}^{T} \int_{S^{2}} \int_{S^{2}}\left(e^{-\frac{|(s+\lambda h) \xi+(s+h) \eta|^{2}}{8 w}}\right. \\
& \left.+e^{-\frac{|(s+h)(\xi+\eta)|^{2}}{4 w}}+e^{-\frac{|s \xi+(s+h) \eta|^{2}}{4 w}}\right) s \sigma(d \xi) \sigma(d \eta) d s d w d \lambda .
\end{aligned}
$$

We claim that this quantity is bounded by $C h^{\rho_{1}}$ if $0<\rho_{1}<\min (\alpha-1,1)$. To show this claim, we first estimate the quantity

$$
I_{1}:=\int_{0}^{T} \int_{S^{2}} \int_{S^{2}} s e^{-\frac{|(s+\lambda h) \xi+(s+h) \eta|^{2}}{8 w}} \sigma(d \xi) \sigma(d \eta) d s .
$$

Using the Fourier transform (see Lemma 6.5), the change of variables $\xi \sqrt{w}=\eta$, and taking $0<\varepsilon<1$ we obtain

$$
\begin{aligned}
I_{1} & =C \int_{0}^{T} \int_{\mathbb{R}^{3}} \frac{s}{(s+h)(s+\lambda h)} w^{\frac{3}{2}} e^{-2 w|\xi|^{2}} \frac{\sin (s+h)|\xi|}{|\xi|} \frac{\sin (s+\lambda h)|\xi|}{|\xi|} d \xi d s \\
& \leq C w^{\frac{3}{2}} \int_{0}^{T} s(s+h)^{\varepsilon-1}(s+\lambda h)^{\varepsilon-1} d s \int_{\mathbb{R}^{3}} e^{-2 w|\xi|^{2}}|\xi|^{2 \varepsilon-2} d \xi \\
& \leq C w^{1-\varepsilon} \int_{\mathbb{R}^{3}} e^{-2|\eta|^{2}}|\eta|^{2 \varepsilon-2} d \eta \leq C w^{1-\varepsilon} .
\end{aligned}
$$


Similarly, we have

$$
\int_{0}^{T} \int_{S^{2}} \int_{S^{2}} s e^{-\frac{|(s+h) \xi+(s+h) \eta|^{2}}{4 w}} \sigma(d \xi) \sigma(d \eta) d s \leq C w^{1-\varepsilon}
$$

and

$$
\int_{0}^{T} \int_{S^{2}} \int_{S^{2}} s e^{-\frac{|s \xi+(s+h) \eta|^{2}}{4 w}} \sigma(d \xi) \sigma(d \eta) d s \leq C w^{1-\varepsilon} .
$$

Therefore,

$$
\begin{aligned}
& \sup _{0<h \leq 1} \int_{0}^{1} \int_{0}^{\infty} w^{\frac{\alpha-5-\rho_{1}}{2}} e^{-w} \int_{0}^{T} \int_{S^{2}} \int_{S^{2}}\left(e^{-\frac{|(s+\lambda h) \xi+(s+h) \eta|^{2}}{8 w}}\right. \\
& \left.\quad+e^{-\frac{|(s+h)(\xi+\eta)|^{2}}{4 w}}+e^{-\frac{|s \xi+(s+h) \eta|^{2}}{4 w}}\right) \times s \sigma(d \xi) \sigma(d \eta) d s d w d \lambda \\
& \leq C \int_{0}^{1} \int_{0}^{\infty} w^{\frac{\alpha-5-\rho_{1}}{2}+1-\varepsilon} e^{-w} d w d \lambda<\infty,
\end{aligned}
$$

and (4.1) is satisfied with $0<\rho_{1}<\min (\alpha-1,1)$ ).

To check condition (4.2), we note that

$$
\begin{aligned}
& \left|e^{-\frac{|x+h \xi+h \eta|^{2}}{4 w}}-e^{-\frac{|x+h \xi|^{2}}{4 w}}-e^{-\frac{|x+h \eta|^{2}}{4 w}}+e^{-\frac{|x|^{2}}{4 w}}\right|=\left|\int_{0}^{1} \int_{0}^{1} \frac{\partial^{2}}{\partial \lambda \partial \mu} e^{-\frac{|x+\lambda h \xi+\mu h \eta|^{2}}{4 w}} d \lambda d \mu\right| \\
& =\mid \int_{0}^{1} \int_{0}^{1} e^{-\frac{|x+\lambda h \xi+\mu h \eta|^{2}}{4 w}}\left(\frac{1}{4 w^{2}}\langle h \xi, x+\lambda h \xi+\mu h \eta\rangle\langle h \eta, x+\lambda h \xi+\mu h \eta\rangle\right. \\
& \left.\quad-\frac{1}{2 w}\langle h \eta, h \xi\rangle\right) d \lambda d \mu \mid \\
& \leq \int_{0}^{1} \int_{0}^{1}\left(e^{-\frac{|x+\lambda h \xi+\mu h \eta|^{2}}{4 w}} \frac{1}{4 w^{2}}|x+\lambda h \xi+\mu h \eta|^{2} h^{2}+e^{-\frac{|x+\lambda h \xi+\mu h \eta|^{2}}{4 w}} \frac{1}{2 w} h^{2}\right) d \lambda d \mu \\
& \leq C \int_{0}^{1} \int_{0}^{1} e^{-\frac{|x+\lambda h \xi+\mu h|^{2}}{8 w}} \frac{h^{2}}{w} d \lambda d \mu .
\end{aligned}
$$


By considering the cases $\frac{h^{2}}{w} \leq 1$ and $\frac{h^{2}}{w}>1$, we have for any $0 \leq \rho_{2} \leq 2$,

$$
\begin{aligned}
& \left|e^{-\frac{|x+h \xi+h \eta|^{2}}{4 w}}-e^{-\frac{|x+h \xi|^{2}}{4 w}}-e^{-\frac{|x+h \eta|^{2}}{4 w}}+e^{-\frac{|x|^{2}}{4 w}}\right| \\
& \leq C\left(\frac{h^{2}}{w}\right)^{\frac{\rho_{2}}{2}} \int_{0}^{1} \int_{0}^{1}\left(e^{-\frac{|x+\lambda h \xi+\mu h \eta|^{2}}{8 w}}+e^{-\frac{|x+h \xi+h \eta|^{2}}{4 w}}+e^{-\frac{|x+h \xi|^{2}}{4 w}}\right. \\
& \left.\quad+e^{-\frac{|x+h \eta|^{2}}{4 w}}+e^{-\frac{|x|^{2}}{4 w}}\right) d \lambda d \mu \\
& :=C h^{\rho_{2}} w^{-\frac{\rho 2}{2}} q_{h, \xi, \eta}(x, w) .
\end{aligned}
$$

Therefore, we obtain

$$
\begin{aligned}
& |f(x+h \xi+h \eta)-f(x+h \xi)-f(x+h \eta)+f(x)| \\
& =C h^{\rho_{2}} \int_{0}^{\infty} w^{\frac{\alpha-5-\rho_{2}}{2}} e^{-w} q_{h, \xi, \eta}(x, w) d w,
\end{aligned}
$$

and

$$
\begin{aligned}
& \int_{0}^{T} \int_{S^{2}} \int_{S^{2}} \mid f(s(\xi+\eta)+h(\xi+\eta))-f(s(\xi+\eta)+h \xi) \\
& \quad-f(s(\xi+\eta)+h \eta)+f(s(\xi+\eta)) \mid \times s^{2} \sigma(d \xi) \sigma(d \eta) d s \\
& \leq C h^{\rho_{2}} \int_{0}^{1} \int_{0}^{1} \int_{0}^{\infty} w^{\frac{\alpha-5-\rho_{2}}{2}} e^{-w} \int_{0}^{T} \int_{S^{2}} \int_{S^{2}}\left(e^{-\frac{|(s+\lambda h) \xi+(s+\mu h) \eta|^{2}}{8 w}}\right. \\
& \quad+e^{-\frac{|(s+h)(\xi+\eta)|^{2}}{4 w}}+e^{-\frac{|(s+h) \xi+s \eta|}{4 w}}+e^{-\frac{\mid s \xi+(s+h) \eta}{4 w}} \\
& \left.+e^{-\frac{\mid s \xi+s \eta}{4 w}}\right) s^{2} \sigma(d \xi) \sigma(d \eta) d s d w d \lambda d \mu
\end{aligned}
$$

We claim that when $0<\rho_{2}<\min (\alpha-1,2)$, the above expression is bounded by $h^{\frac{\rho_{2}}{2}}$. To show this claim, we first estimate the integral

$$
I_{2}:=\int_{0}^{T} \int_{S^{2}} \int_{S^{2}} s^{2} e^{-\frac{|(s+\lambda h) \xi+(s+\mu h) \eta|}{8 w}} \sigma(d \xi) \sigma(d \eta) d s .
$$


Using the Fourier transform (see Lemma 6.5) and the change of variable $\sqrt{w} \xi=\eta$, we obtain

$$
\begin{aligned}
I_{2} & =\int_{0}^{T} \frac{s^{2}}{(s+\lambda h)(s+\mu h)} \int_{\mathbb{R}^{3}} w^{\frac{3}{2}} e^{-2 w|\xi|^{2}} \frac{\sin (s+\lambda h)|\xi|}{|\xi|} \frac{\sin (s+\mu h)|\xi|}{|\xi|} d \xi d s \\
& \leq C \int_{\mathbb{R}^{3}} e^{-2|\eta|^{2}} \frac{w}{|\eta|^{2}} d \eta \leq C w,
\end{aligned}
$$

where the constant $C$ does not depend on $\lambda$ and $\mu$. The same estimation can be done for each of the other integrals and we obtain

$$
\begin{aligned}
& \iiint_{0}^{T} \int_{S^{2} S^{2}} s^{2}\left(e^{-\frac{|(s+h)(\xi+\eta)|^{2}}{4 w}}+e^{-\frac{|(s+h) \xi+s \eta|^{2}}{4 w}}+e^{-\frac{|s \xi+(s+h) \eta|^{2}}{4 w}}+e^{-\frac{|s \xi+s \eta|^{2}}{4 w}}\right) \\
& \quad \times \sigma(d \xi) \sigma(d \eta) d s \leq C w .
\end{aligned}
$$

Thus,

$$
\begin{aligned}
& \int_{0}^{T} \int_{S^{2}} \int_{S^{2}} \mid f(s(\xi+\eta)+h(\xi+\eta))-f(s(\xi+\eta)+h \xi) \\
& \quad-f(s(\xi+\eta)+h \eta)+f(s(\xi+\eta)) \mid \times s^{2} \sigma(d \xi) \sigma(d \eta) d s \\
& \leq C h^{\rho_{2}} \int_{0}^{\infty} w^{\frac{\alpha-3-\rho_{2}}{2}} e^{-w} d w \leq C h^{\rho_{2}},
\end{aligned}
$$

and the (4.2) is satisfied for $0<\rho_{2}<\min (\alpha-1,2)$. This completes the proof.

Notice that, with the notation of Corollary 4.2, for the Bessel kernel we can take $\kappa_{1}=\kappa_{2}<(\alpha-1) \wedge 1$, and we deduce the local Hölder continuity of the solution $u$ in space and time variables of order $\kappa<\min \left(\gamma_{1}, \gamma_{2}, \frac{\alpha-1}{2} \wedge 1\right)$.

\section{The fractional noise}

In this section we consider the case where $\dot{W}(t, x)$ is fractional Brownian noise in the space variable with Hurst parameters $H_{1}, H_{2}, H_{3}$ in each direction. That is, suppose that $\left\{W(t, x), t \geq 0, x \in \mathbb{R}^{3}\right\}$ is a centered Gaussian field with the covariance

$$
\mathbb{E}[W(s, x) W(t, y)]=(s \wedge t) \prod_{i=1}^{3} R_{i}\left(x_{i}, y_{i}\right), \quad s, t \geq 0, x, y \in \mathbb{R}^{3},
$$


where $x=\left(x_{1}, x_{2}, x_{3}\right), y=\left(y_{1}, y_{2}, y_{3}\right)$ and

$$
R_{i}(u, v)=\frac{1}{2}\left(|u|^{2 H_{i}}+|v|^{2 H_{i}}-|u-v|^{2 H_{i}}\right) .
$$

Then $\dot{W}(t, x)$ is the formal partial derivative $\frac{\partial^{4} W}{\partial t \partial x_{1} \partial x_{2} \partial x_{3}}(t, x)$. We will require $\frac{1}{2}<H_{i}<1, i=1,2,3$. This choice of noise corresponds to the covariance function

$$
f(x)=c_{H}\left|x_{1}\right|^{2 H_{1}-2}\left|x_{2}\right|^{2 H_{2}-2}\left|x_{3}\right|^{2 H_{3}-2},
$$

where $H=\left(H_{1}, H_{2}, H_{3}\right)$ and $c_{H}=\prod_{i=1}^{3} H_{i}\left(2 H_{i}-1\right)$. Here and in what follows for simplicity, we omit the coefficient $c_{H}$ in the expression of $f(x)$. The corresponding spectral measure is

$$
\mu(d \xi)=C_{H}\left|\xi_{1}\right|^{1-2 H_{1}}\left|\xi_{2}\right|^{1-2 H_{2}}\left|\xi_{3}\right|^{1-2 H_{3}}
$$

for some constant $C_{H}$ which depends only on $H$. We will apply Theorems 3.2 and 4.1 to get the Hölder continuity of the solution to Eq. (1.1) in the space and time variables.

Theorem 6.1 Assume conditions $(a)$ and $(b)$ in Theorem 3.1 and let $f$ be given by (6.3) (without the constant $c_{H}$ ) with $\mathrm{H}_{1}+\mathrm{H}_{2}+\mathrm{H}_{3}>2$. Set

$$
\bar{\kappa}=H_{1}+H_{2}+H_{3}-2,
$$

and choose constants $\kappa_{i}>0, i=0,1,2,3$ such that $\kappa_{i}<\min \left(H_{i}-\frac{1}{2}, \bar{\kappa}, \gamma_{1}, \gamma_{2}\right)$ for $i=1,2,3$, and $\kappa_{0} \leq \min \left(\kappa_{1}, \kappa_{2}, \kappa_{3}\right)$. Then the solution to (1.1) is locally Hölder continuous with exponent $\kappa_{0}$ in the time variable and with exponent $\kappa_{i}$ in the ith direction. Namely, for any bounded rectangle $I \subset \mathbb{R}^{3}$, there exists a random variable $K$ (depending on I and the constants $\kappa_{i}$ 's), such that

$$
|u(t, x)-u(\bar{t}, y)| \leq K\left(\left|x_{1}-y_{1}\right|^{\kappa_{1}}+\left|x_{2}-y_{2}\right|^{\kappa_{2}}+\left|x_{3}-y_{3}\right|^{\kappa_{3}}+|\bar{t}-t|^{\kappa_{0}}\right)
$$

for all $t, \bar{t} \in[0, T], x, y \in I$.

Proof First we consider the space variable. Proceeding as in the proof of Theorem 3.2 , it is easy to see that if for some number $0<\gamma \leq 1, \mathcal{F}\left(\left|\xi_{1}\right|^{2 \gamma} \mu(d \xi)\right)(w)$ is a nonnegative locally integrable function and

$$
\int_{\mathbb{R}^{3}} \frac{\left|\xi_{1}\right|^{2 \gamma} \mu(d \xi)}{1+|\xi|^{2}}<\infty
$$

then if $\kappa_{1}=\min \left(\gamma, \gamma_{1}, \gamma_{2}\right)$, for any bounded rectangle $I \subset \mathbb{R}^{3}$, and for any $q \geq 2$, there exists a constant $C$ such that

$$
\mathbb{E}\left|u\left(t, x_{1}, x_{2}, x_{3}\right)-u\left(t, y_{1}, x_{2}, x_{3}\right)\right|^{q} \leq C\left|x_{1}-y_{1}\right|^{q \kappa_{1}}
$$


for any $t \in[0, T]$ and $x, y \in I$.

We claim that for $0<\gamma<\min \left(H_{1}-\frac{1}{2}, \bar{\kappa}\right), \mathcal{F}\left(\left|\xi_{1}\right|^{2 \gamma} \mu(d \xi)\right)(w)$ is a nonnegative locally integrable function and (6.6) holds. Indeed, since $\mu(d \xi)=\left|\xi_{1}\right|^{1-2 H_{1}}\left|\xi_{2}\right|^{1-2 H_{2}}\left|\xi_{3}\right|^{1-2 H_{3}} d \xi$, we have

$$
\begin{aligned}
\mathcal{F}\left(\left|\xi_{1}\right|^{2 \gamma} \mu(d \xi)\right)(w) & =\mathcal{F}\left(\left|\xi_{1}\right|^{1-2 H_{1}+2 \gamma}\left|\xi_{2}\right|^{1-2 H_{2}}\left|\xi_{3}\right|^{1-2 H_{3}}\right)(w) \\
& =C\left|w_{1}\right|^{-2+2 H_{1}-2 \gamma}\left|w_{2}\right|^{2 H_{2}-2}\left|w_{3}\right|^{2 H_{3}-2}
\end{aligned}
$$

which is well defined because $\gamma<H_{1}-\frac{1}{2}$. To show (6.6), we have

$$
\begin{aligned}
\int_{\mathbb{R}^{3}} \frac{\left|\xi_{1}\right|^{2 \gamma} \mu(d \xi)}{1+|\xi|^{2}} & =\int_{\mathbb{R}^{3}} \frac{\left|\xi_{1}\right|^{1-2\left(H_{1}-\gamma\right)}\left|\xi_{2}\right|^{1-2 H_{2}}\left|\xi_{3}\right|^{1-2 H_{3}}}{1+|\xi|^{2}} d \xi \\
& \leq \int_{\mathbb{R}} \frac{\left|\xi_{1}\right|^{1-2\left(H_{1}-\gamma\right)}}{\left(1+\left|\xi_{1}\right|^{2}\right)^{\alpha_{1}}} d \xi_{1} \int_{\mathbb{R}} \frac{\left|\xi_{2}\right|^{1-2 H_{2}}}{\left(1+\left|\xi_{2}\right|^{2}\right)^{\alpha_{2}}} d \xi_{2} \int_{\mathbb{R}} \frac{\left|\xi_{3}\right|^{1-2 H_{3}}}{\left(1+\left|\xi_{3}\right|^{2}\right)^{\alpha_{3}}} d \xi_{3},
\end{aligned}
$$

where the $\alpha_{i}$ 's are positive with $\alpha_{1}+\alpha_{2}+\alpha_{3}=1$. When $1-2\left(H_{1}-\gamma\right)-2 \alpha_{1}<-1$, $1-2 H_{2}-2 \alpha_{2}<-1$ and $1-2 H_{3}-2 \alpha_{3}<-1$, the above three integrals are finite. It is elementary to see such $\alpha_{i}$ 's exist under the condition $\gamma<H_{1}+H_{2}+H_{3}-2$. The same argument holds for the other coordinates.

For the time variable, we will check conditions (1) and (3) in Theorem 4.1. To see that condition (1) in Theorem 4.1 is satisfied for some $0<v \leq 1$, take positive numbers $\varepsilon_{i}, i=1,2,3$ such that $\varepsilon_{1}+\varepsilon_{2}+\varepsilon_{3}=1$ and $2 H_{i}-1-\varepsilon_{i}>0$ for $i=1,2,3$. Then we have

$$
\begin{aligned}
\int_{|z| \leq h} \frac{f(z)}{|z|} d z & \leq \int_{\left|z_{1}\right| \leq h} \frac{\left|z_{1}\right|^{2 H_{1}-2}}{\left|z_{1}\right|^{\varepsilon_{1}}} d z_{1} \int_{\left|z_{2}\right| \leq h} \frac{\left|z_{2}\right|^{2 H_{2}-2}}{\left|z_{2}\right|^{\varepsilon_{2}}} d z_{2} \int_{\left|z_{3}\right| \leq h} \frac{\left|z_{3}\right|^{2 H_{3}-2}}{\left|z_{3}\right|^{\varepsilon_{3}}} d z_{3} \\
& \leq C h^{2 H_{1}-1-\varepsilon_{1}} h^{2 H_{2}-1-\varepsilon_{2}} h^{2 H_{3}-1-\varepsilon_{3}}=h^{2\left(H_{1}+H_{2}+H_{3}-2\right)} .
\end{aligned}
$$

So condition (1) in Theorem 4.1 is satisfied with $v=\min \left(2\left(H_{1}+H_{2}+H_{3}-2\right), 1\right)$.

To check (4.1), let $x=a(\xi+\eta)+h \eta$. Then we decompose the difference $f(x+$ $h \xi)-f(x)$ into the sum of three terms, each of them containing an increment in one direction, and we obtain

$$
\begin{aligned}
& |f(x+h \xi)-f(x)| \\
& =|| x_{1}+\left.h \xi_{1}\right|^{2 H_{1}-2}\left|x_{2}+h \xi_{2}\right|^{2 H_{2}-2}\left|x_{3}+h \xi_{3}\right|^{2 H_{3}-2}-\left|x_{1}\right|^{2 H_{1}-2}\left|x_{2}\right|^{2 H_{2}-2}\left|x_{3}\right|^{2 H_{3}-2} \mid \\
& \leq|| x_{1}+\left.h \xi_{1}\right|^{2 H_{1}-2}-\left|x_{1}\right|^{2 H_{1}-2}|| x_{2}+\left.h \xi_{2}\right|^{2 H_{2}-2}\left|x_{3}+h \xi_{3}\right|^{2 H_{3}-2} \\
& \quad+\left|x_{1}\right|^{2 H_{1}-2}|| x_{2}+\left.h \xi_{2}\right|^{2 H_{2}-2}-\left|x_{2}\right|^{2 H_{2}-2}|| x_{3}+\left.h \xi_{3}\right|^{2 H_{3}-2} \\
& \quad+\left|x_{1}\right|^{2 H_{1}-2}\left|x_{2}\right|^{2 H_{2}-2}|| x_{3}+\left.h \xi_{3}\right|^{2 H_{3}-2}-\left|x_{3}\right|^{2 H_{3}-2} \mid .
\end{aligned}
$$


We claim that for some $\rho_{1} \in(0,1]$, the integral on $[0, T] \times S^{2} \times S^{2}$ of each of these three terms with respect to the measure $s \sigma(d \xi) \sigma(d \eta) d s$ is bounded by $C h^{\rho_{1}}$. To show this claim, we apply (5.1) with $d=1, b=\rho_{1}<\min \left(2 H_{1}-1,2 H_{2}-\right.$ $\left.1,2 H_{3}-1,2\left(H_{1}+H_{2}+H_{3}-2\right)\right), a=2 H_{i}-\rho_{1}-1$ and $u=x_{i}$ to the $i$ th summand $(i=1,2,3)$ and we get

$$
\begin{aligned}
& |f(x+h \xi)-f(x)| \\
& \leq h^{\rho_{1}} \int_{\mathbb{R}} d w\left|x_{1}-h w\right|^{2 H_{1}-2-\rho_{1}}|| w+\left.\xi_{1}\right|^{\rho_{1}-1}-|w|^{\rho_{1}-1}|| x_{2}+\left.h \xi_{2}\right|^{2 H_{2}-2}\left|x_{3}+h \xi_{3}\right|^{2 H_{3}-2} \\
& \quad+h^{\rho_{1}} \int_{\mathbb{R}} d w\left|x_{2}-h w\right|^{2 H_{2}-2-\rho_{1}}|| w+\left.\xi_{2}\right|^{\rho_{1}-1}-\left.|w|^{\rho_{1}-1}|| x_{1}\right|^{2 H_{1}-2}\left|x_{3}+h \xi_{3}\right|^{2 H_{3}-2} \\
& \quad+h^{\rho_{1}} \int_{\mathbb{R}} d w\left|x_{3}-h w\right|^{2 H_{3}-2-\rho_{1}}|| w+\left.\xi_{3}\right|^{\rho_{1}-1}-\left.|w|^{\rho_{1}-1}|| x_{1}\right|^{2 H_{1}-2}\left|x_{2}\right|^{2 H_{2}-2} \\
& :=h^{\rho_{1}}\left(g_{h, \xi}^{1}(x)+g_{h, \xi}^{2}(x)+g_{h, \xi}^{3}(x)\right) .
\end{aligned}
$$

We want to show that for $i=1,2,3$

$$
\sup _{0<h \leq 1} \int_{0}^{T} \int_{S^{2} \times S^{2}} s g_{h, \xi}^{i}(s \xi+(s+h) \eta) \sigma(d \xi) \sigma(d \eta) d s<\infty
$$

We will consider only the case $i=1$, the other two terms being similar. By splitting the integral with respect to $w$ into two parts, one over $|w| \leq 3$, and another one over $|w|>3$, just as we did for the Riesz kernel, we have

$$
\begin{aligned}
& \int_{0} \int_{S^{2} \times S^{2}} s g_{h, \xi}^{1}(s \xi+(s+h) \eta) \sigma(d \xi) \sigma(d \eta) d s \\
& \leq \int_{0}^{T} \int_{S^{2} \times S^{2}} s \int_{|w| \leq 3}\left|s \xi_{1}+(s+h) \eta_{1}-h w\right|^{2 H_{1}-2-\rho_{1}}\left|(s+h) \xi_{2}+(s+h) \eta_{2}\right|^{2 H_{2}-2} \\
& \quad \times\left|(s+h) \xi_{3}+(s+h) \eta_{3}\right|^{2 H_{3}-2}\left|w+\xi_{1}\right|^{\rho_{1}-1} d w \sigma(d \xi) \sigma(d \eta) d s \\
& \quad+\int_{0}^{T} \int_{S^{2} \times S^{2}} s \int_{|w| \leq 3}\left|s \xi_{1}+(s+h) \eta_{1}-h w\right|^{2 H_{1}-2-\rho_{1}}\left|(s+h) \xi_{2}+(s+h) \eta_{2}\right|^{2 H_{2}-2} \\
& \quad \times\left|(s+h) \xi_{3}+(s+h) \eta_{3}\right|^{2 H_{3}-2}|w|^{\rho_{1}-1} d w \sigma(d \xi) \sigma(d \eta) d s
\end{aligned}
$$




$$
\begin{aligned}
& +\int_{0}^{T} \int_{S^{2} \times S^{2}} s \int_{|w|>3}\left|s \xi_{1}+(s+h) \eta_{1}-h w\right|^{2 H_{1}-2-\rho_{1}}\left|(s+h) \xi_{2}+(s+h) \eta_{2}\right|^{2 H_{2}-2} \\
& \times\left|(s+h) \xi_{3}+(s+h) \eta_{3}\right|^{2 H_{3}-2}|| w+\left.\xi_{1}\right|^{\rho_{1}-1}-|w|^{\rho_{1}-1} \mid d w \sigma(d \xi) \sigma(d \eta) d s \\
& :=I_{1}+I_{2}+I_{3} .
\end{aligned}
$$

For integral $I_{1}$, using the change of variable $w+\xi_{1} \rightarrow w$ and the Fourier transform, we can write

$$
\begin{aligned}
I_{1} \leq & \int_{0}^{T} \int_{S^{2} \times S^{2}} s \int_{|w| \leq 4}\left|(s+h) \xi_{1}+(s+h) \eta_{1}-h w\right|^{2 H_{1}-2-\rho_{1}} \mid(s+h) \xi_{2} \\
& +\left.(s+h) \eta_{2}\right|^{2 H_{2}-2} \\
& \times\left|(s+h) \xi_{3}+(s+h) \eta_{3}\right|^{2 H_{3}-2}|w|^{\rho_{1}-1} d w \sigma(d \eta) \sigma(d \xi) d s \\
\leq & \int_{0}^{T} \int_{|w| \leq 4} d w|w|^{\rho_{1}-1} \sup _{w \in \mathbb{R}} \int_{\mathbb{R}^{3}} \frac{s}{(s+h)^{2}}\left|z_{1}\right|^{1-2 H_{1}+\rho_{1}} e^{i z_{1} h w}\left|z_{2}\right|^{1-2 H_{2}}\left|z_{3}\right|^{1-2 H_{3}} \\
& \times\left(\frac{\sin (s+h)|z|}{|z|}\right)^{2} d z d s .
\end{aligned}
$$

By the change of variable $(s+h) z=x$, the bound $\left|e^{i z_{1} h w}\right| \leq 1$ and direct calculation, we see the integral is finite uniformly in $0<h \leq 1$.

For the integral $I_{2}$ we can write

$$
\begin{aligned}
I_{2}= & \int_{|w| \leq 3} \int_{0}^{T} \int_{S^{2} \times S^{2}} s\left|s \xi_{1}+(s+h) \eta_{1}-h w\right|^{2 H_{1}-2-\rho_{1}}\left|(s+h) \xi_{2}+(s+h) \eta_{2}\right|^{2 H_{2}-2} \\
& \times\left|(s+h) \xi_{3}+(s+h) \eta_{3}\right|^{2 H_{3}-2} \sigma(d \xi) \sigma(d \eta) d s|w|^{\rho_{1}-1} d w \\
= & \int_{|w| \leq 3} \int_{0}^{T} \int_{\mathbb{R}^{3} \times \mathbb{R}^{3}} \frac{s}{(s+h)^{2}} G(s+h, d y) G(s+h, d z) \\
& \times\left|\frac{s}{s+h} y_{1}+z_{1}-h w\right|^{2 H_{1}-2-\rho_{1}}\left|y_{2}+z_{2}\right|^{2 H_{2}-2}\left|y_{3}+z_{3}\right|^{2 H_{3}-2} d s|w|^{\rho_{1}-1} d w \\
= & \int_{|w| \leq 3} \int_{0}^{T} \frac{s}{(s+h)^{2}} G(s+h) * G^{\psi}{ }_{\mathbb{R}^{3}}(s+h)(z) \\
& \times\left|z_{1}-h w\right|^{2 H_{1}-2-\rho_{1}}\left|z_{2}\right|^{2 H_{2}-2}\left|z_{3}\right|^{2 H_{3}-2} d z d s|w|^{\rho_{1}-1} d w,
\end{aligned}
$$


where $\psi(y)=\left(\frac{s}{s+h} y_{1}, y_{2}, y_{3}\right)$, and $G^{\psi}(s+h)$ denotes the image of the measure $G(s+h)$ by the mapping $\psi$. Then using the Fourier transform we obtain

$$
\begin{aligned}
& I_{2}=\int_{|w| \leq 3} \int_{0}^{T} \int_{\mathbb{R}^{3}} \frac{s}{(s+h)^{2}}\left(\mathcal{F}\left(G(s+h) * G^{\psi}(s+h)\right)\right)(\xi) \\
& \times e^{i \xi_{1} w}\left|\xi_{1}\right|^{1+\rho_{1}-2 H_{1}}\left|\xi_{2}\right|^{1-2 H_{2}}\left|\xi_{3}\right|^{1-2 H_{3}} d \xi d s|w|^{\rho_{1}-1} d w \\
& \leq \int_{|w| \leq 3} \int_{0}^{T} \int_{\mathbb{R}^{3}} \frac{s}{(s+h)^{2}}\left|\frac{\sin (s+h)|\xi|}{|\xi|}\right|\left|\frac{\sin (s+h)\left|\left(\frac{s}{s+h} \xi_{1}, \xi_{2}, \xi_{3}\right)\right|}{\left|\left(\frac{s}{s+h} \xi_{1}, \xi_{2}, \xi_{3}\right)\right|}\right| \\
& \times\left|\xi_{1}\right|^{1+\rho_{1}-2 H_{1}}\left|\xi_{2}\right|^{1-2 H_{2}}\left|\xi_{3}\right|^{1-2 H_{3}} d \xi d s|w|^{\rho_{1}-1} d w \\
& \leq \int_{|w| \leq 3} \int_{0}^{T} \int_{|\xi| \leq 1}\left|\xi_{1}\right|^{1+\rho_{1}-2 H_{1}}\left|\xi_{2}\right|^{1-2 H_{2}}\left|\xi_{3}\right|^{1-2 H_{3}} d \xi d s|w|^{\rho_{1}-1} d w \\
& +\int_{|w| \leq 3} \int_{0}^{T} \int_{|\xi|>1} \frac{s}{(s+h)^{2}}\left|\frac{\sin (s+h)|\xi|}{|\xi|}\right| \\
& \times \frac{1}{\frac{s}{s+h}|\xi|}\left|\xi_{1}\right|^{1+\rho_{1}-2 H_{1}}\left|\xi_{2}\right|^{1-2 H_{2}}\left|\xi_{3}\right|^{1-2 H_{3}} d \xi d s|w|^{\rho_{1}-1} d w<\infty,
\end{aligned}
$$

where in the first inequality above we used the fact that $\left|e^{-i \xi_{1} w}\right| \leq 1$, and in the last inequality we used that fact that $|\sin x| \leq|x|^{\varepsilon}$ for any $\varepsilon>0$. The above integral is finite uniformly in $w$ and $0<h \leq 1$.

For the third integral $I_{3}$, we can bound ||$w+\left.\xi_{1}\right|^{\rho_{1}-1}-|w|^{\rho_{1}-1} \mid$ by $C|w|^{\rho_{1}-2}$ as in the example of the Riesz kernel, and proceed as in the second integral $I_{2}$. Applying the same argument for the other two terms, we get (6.8) with $\rho_{1} \in\left(0, \min \left(2 H_{1}-\right.\right.$ $\left.1,2 H_{2}-1,2 H_{3}-1,2 \bar{\kappa}\right)$. Therefore, condition (4.1) is satisfied with $0<\rho_{1}<$ $\min \left(2 H_{1}-1,2 H_{2}-1,2 H_{3}-1,2 \bar{\kappa}\right)$.

For condition (4.2), we use the inequality

$$
\begin{aligned}
& |f((s+h) \xi+(s+h) \eta)-f((s+h) \xi+s \eta)-f((s+h) \eta+s \xi)+f(s(\xi+\eta))| \\
& \leq|f((s+h) \xi+(s+h) \eta)-f((s+h) \xi+s \eta)|+|f((s+h) \eta+s \xi)-f(s \xi+s \eta)| .
\end{aligned}
$$

Then we can apply the previous procedure to both terms on the right-hand side and the argument is the same as in the case of condition (4.1). We conclude that (4.2) is satisfied with $0<\rho_{2}<\min \left(2 \mathrm{H}_{1}-1,2 \mathrm{H}_{2}-1,2 \mathrm{H}_{3}-1,2 \bar{\kappa}\right)$.

In summary, we can take $v=\min (2 \bar{\kappa}, 1)$ and $\rho_{1}=\rho_{2} \in\left(0, \min \left(2 H_{1}-1,2 H_{2}-\right.\right.$ $\left.1,2 H_{3}-1,2 \bar{\kappa}\right)$ ), and Theorem 4.2 together with the moment estimate (6.7) leads to the desired Hölder continuity in the space and time variables via an application of Kolmogorov's continuity theorem.

Consider Eq. (1.1) with vanishing initial conditions $v_{0}, \bar{v}_{0}$ and coefficients $\sigma \equiv 1$ and $b \equiv 0$. That means, we consider the stochastic wave equation with additive fractional noise 


$$
\left\{\begin{array}{l}
\left(\frac{\partial^{2}}{\partial t^{2}}-\Delta u\right)(t, x)=\dot{W}(t, x), \\
u(0, x)=\frac{\partial u}{\partial t}(0, x)=0 .
\end{array}\right.
$$

The covariance function of the noise is given by (6.3) with $H_{i}>\frac{1}{2}$ for $i=1,2,3$ and recall that $\bar{\kappa}=H_{1}+H_{2}+H_{3}-2>0$.

For this equation the solution can be written as

$$
u(t, x)=\int_{0}^{t} \int_{\mathbb{R}^{3}} G(t-s, x-y) W(d s, d y) .
$$

In this case we are going to show that $\bar{\kappa}$ is the optimal exponent for the Hölder continuity of the solution $u$ in the space and time variables.

Theorem 6.2 Let u be the solution to the stochastic partial differential equation (6.9). Then

(a) There are two positive constants $c_{1}$ and $c_{2}$ such that

$$
c_{1}|x-y|^{2 \bar{\kappa}} \leq \mathbb{E}\left(|u(t, x)-u(t, y)|^{2}\right) \leq c_{2}|x-y|^{2 \bar{\kappa}}
$$

for all $x, y \in \mathbb{R}^{3}$ and $t \in[0, T]$.

(b) For any fixed $t_{0} \in(0, T]$ there are two positive constants $c_{1}$ and $c_{2}$ such that

$$
c_{1}|\bar{t}-t|^{2 \bar{\kappa}} \leq \mathbb{E}\left(|u(t, x)-u(\bar{t}, x)|^{2}\right) \leq c_{2}|\bar{t}-t|^{2 \bar{\kappa}}
$$

for all $t, \bar{t} \in\left[t_{0}, T\right]$ and $x \in \mathbb{R}^{3}$.

Proof For any $x \in \mathbb{R}^{3}$, set $R(x)=\mathbb{E}(u(t, x) u(t, 0))$. It is easy to see that

$$
\mathbb{E}\left(|u(t, x)-u(t, y)|^{2}\right)=2(R(0)-R(x-y)) .
$$

Without loss of generality, we may assume that $t=1$ and $y=0$. We have by Lemma 6.5

$$
\begin{aligned}
R(0)-R(x)= & \int_{0}^{1} d s \int_{\mathbb{R}^{3}} \mu(d \xi)\left(1-e^{i \xi \cdot x}\right)|\mathcal{F} G(1-s)(\xi)|^{2} \\
= & \frac{1}{2} \int_{\mathbb{R}^{3}} d \xi\left|\xi_{1}\right|^{1-2 H_{1}}\left|\xi_{2}\right|^{1-2 H_{2}}\left|\xi_{3}\right|^{1-2 H_{3}} \frac{1}{|\xi|^{2}}(1-\cos (\xi \cdot x)) \\
& \times\left(1-\frac{\sin (2|\xi|)}{2|\xi|}\right) .
\end{aligned}
$$


The integrand is non-negative. For clarity we may assume that $\left|x_{1}\right| \leq\left|x_{2}\right| \leq\left|x_{3}\right|$. If $\left|\xi_{3}\right| \geq 1$, then $1-\frac{\sin (2|\xi|)}{2|\xi|} \geq \frac{1}{2}$. Thus using the change of variable $\xi x_{3}=\eta$, we have

$$
\begin{aligned}
& R(0)-R(x) \\
& \geq \frac{1}{4} \int_{\left|\xi_{3}\right| \geq 1}\left|\xi_{1}\right|^{1-2 H_{1}}\left|\xi_{2}\right|^{1-2 H_{2}}\left|\xi_{3}\right|^{1-2 H_{3}}(1-\cos (\xi \cdot x)) \frac{1}{|\xi|^{2}} d \xi \\
& =\frac{1}{4}\left|x_{3}\right|^{2 \kappa} \int_{\left|\eta_{3}\right| \geq\left|x_{3}\right|}\left|\eta_{1}\right|^{1-2 H_{1}}\left|\eta_{2}\right|^{1-2 H_{2}}\left|\eta_{3}\right|^{1-2 H_{3}}\left[1-\cos \left(\frac{x_{1}}{x_{3}} \eta_{1}+\frac{x_{2}}{x_{3}} \eta_{2}+\eta_{3}\right)\right] \frac{1}{|\eta|^{2}} d \eta .
\end{aligned}
$$

If $x$ is in a bounded interval $I$, then there is $L>0$ such that $\left|x_{3}\right| \leq L$. Thus

$$
\begin{aligned}
& R(0)-R(x) \\
& \geq \frac{1}{4}\left|x_{3}\right|^{2 \kappa} \int_{\left|\eta_{3}\right| \geq L}\left|\eta_{1}\right|^{1-2 H_{1}}\left|\eta_{2}\right|^{1-2 H_{2}}\left|\eta_{3}\right|^{1-2 H_{3}}\left[1-\cos \left(\frac{x_{1}}{x_{3}} \eta_{1}+\frac{x_{2}}{x_{3}} \eta_{2}+\eta_{3}\right)\right] \frac{1}{|\eta|^{2}} d \eta \\
& \geq \frac{1}{4}\left|x_{3}\right|^{2 \kappa} \inf _{\left|u_{1}\right|,\left|u_{2}\right| \leq 1} \int_{\left|\eta_{3}\right| \geq L}\left|\eta_{1}\right|^{1-2 H_{1}}\left|\eta_{2}\right|^{1-2 H_{2}}\left|\eta_{3}\right|^{1-2 H_{3}} \\
& \times\left[1-\cos \left(u_{1} \eta_{1}+u_{2} \eta_{2}+\eta_{3}\right)\right] \frac{1}{|\eta|^{2}} d \eta .
\end{aligned}
$$

It is easy to see that

$$
g\left(u_{1}, u_{2}\right):=\int_{\left|\eta_{3}\right| \geq L}\left|\eta_{1}\right|^{1-2 H_{1}}\left|\eta_{2}\right|^{1-2 H_{2}}\left|\eta_{3}\right|^{1-2 H_{3}}\left[1-\cos \left(u_{1} \eta_{1}+u_{2} \eta_{2}+\eta_{3}\right)\right] \frac{1}{|\eta|^{2}} d \eta
$$

is a continuous function of $u_{1}, u_{2}$ and for any $u_{1}$ and $u_{2}, g\left(u_{1}, u_{2}\right)$ is positive. Thus

$$
\inf _{\left|u_{1}\right|,\left|u_{2}\right| \leq 1} g\left(u_{1}, u_{2}\right)>0
$$

since the infimum is taken on a compact set. This proves the left-hand side inequality in (6.10).

To show the second inequality in (6.10), we can use the triangular inequality, and it suffices to show the inequality for $x=\left(x_{1}, 0,0\right)$. In this case

$$
\begin{aligned}
R(0)-R(x) & \leq \frac{1}{2} \int_{\mathbb{R}^{3}} d \xi\left|\xi_{1}\right|^{1-2 H_{1}}\left|\xi_{2}\right|^{1-2 H_{2}}\left|\xi_{3}\right|^{1-2 H_{3}} \frac{1}{|\xi|^{2}}\left(1-\cos \left(\xi_{1} x_{1}\right)\right) \\
& \leq \frac{1}{2}\left|x_{1}\right|^{2 \kappa} \int_{\mathbb{R}^{3}} d \xi\left|\xi_{1}\right|^{1-2 H_{1}}\left|\xi_{2}\right|^{1-2 H_{2}}\left|\xi_{3}\right|^{1-2 H_{3}} \frac{1}{|\xi|^{2}}\left(1-\cos \left(\xi_{1}\right)\right) \\
& =C\left|x_{1}\right|^{2 \kappa}
\end{aligned}
$$

which is the second inequality of (6.10). Hence, (a) is proved. 
Now we turn to consider (b). Let $0 \leq t<\bar{t} \leq T$. Then we have

$$
\mathbb{E}\left(|u(t, x)-u(\bar{t}, x)|^{2}\right)=2 Z_{1}(t, \bar{t}, x)+2 Z_{2}(t, \bar{t}, x),
$$

where

$$
Z_{1}(t, \bar{t}, x)=\mathbb{E}\left(\int_{t}^{\bar{t}} \int_{\mathbb{R}^{3}} G(\bar{t}-s, x-y) W(d s, d y)\right)^{2}
$$

and

$$
Z_{2}(t, \bar{t}, x)=\mathbb{E}\left(\int_{0}^{t} \int_{\mathbb{R}^{3}}(G(\bar{t}-s, x-y)-G(t-s, x-y)) W(d s, d y)\right)^{2} .
$$

Integrating with respect to the variable $s$ yields

$$
\begin{aligned}
Z_{1}(t, \bar{t}, x) & =\mathbb{E}\left(\int_{t} \int_{\mathbb{R}^{3}} G(\bar{t}-s, x-y) W(d s, d y)\right)^{2} \\
& =C \int_{t}^{\bar{t}} d s \int_{\mathbb{R}^{3}}|\mathcal{F} G(\bar{t}-s)(\xi)|^{2}\left|\xi_{1}\right|^{1-2 H_{1}}\left|\xi_{2}\right|^{1-2 H_{2}}\left|\xi_{3}\right|^{1-2 H_{3}} d \xi \\
& =C \int_{\mathbb{R}^{3}}\left((\bar{t}-t)-\frac{\sin (2(\bar{t}-t)|\xi|)}{2|\xi|}\right) \frac{1}{|\xi|^{2}}\left|\xi_{1}\right|^{1-2 H_{1}}\left|\xi_{2}\right|^{1-2 H_{2}}\left|\xi_{3}\right|^{1-2 H_{3}} d \xi
\end{aligned}
$$

With the change of variable $(\bar{t}-t) \xi \rightarrow \eta$ the last integral becomes

$$
C(\bar{t}-t)^{2 \sum_{i=1}^{3} H_{i}-3} \int_{\mathbb{R}^{3}}\left(1-\frac{\sin (2|\eta|)}{2|\eta|}\right) \frac{1}{|\eta|^{2}}\left|\eta_{1}\right|^{1-2 H_{1}}\left|\eta_{2}\right|^{1-2 H_{2}}\left|\eta_{3}\right|^{1-2 H_{3}} d \eta .
$$

Therefore, we have

$$
c_{1}|\bar{t}-t|^{2 \bar{\kappa}+1} \leq Z_{1}(t, \bar{t}, x) \leq c_{2}|\bar{t}-t|^{2 \bar{\kappa}+1} .
$$

The term $Z_{2}$ is slightly more complicated. A direct integration in the variable $s$ yields

$$
Z_{2}(t, \bar{t}, x)=\int_{0}^{t} d s \int_{\mathbb{R}^{3}} d \xi\left|\xi_{1}\right|^{1-2 H_{1}}\left|\xi_{2}\right|^{1-2 H_{2}}\left|\xi_{3}\right|^{1-2 H_{3}}
$$




$$
\begin{aligned}
& \times \frac{1}{|\xi|^{2}}(\sin ((\bar{t}-s)|\xi|)-\sin ((t-s)|\xi|))^{2} \\
\geq & \int_{|\xi| \geq(\bar{t}-t)^{-1}} d \xi\left|\xi_{1}\right|^{1-2 H_{1}}\left|\xi_{2}\right|^{1-2 H_{2}}\left|\xi_{3}\right|^{1-2 H_{3}} \frac{1}{|\xi|^{2}}(A(t, \bar{t}, \xi)+B(t, \bar{t}, \xi)) \\
= & t I_{1}+I_{2},
\end{aligned}
$$

where

$$
A(t, \bar{t}, \xi)=t(1-\cos ((\bar{t}-t)|\xi|))
$$

and

$$
\begin{gathered}
B(t, \bar{t}, \xi)=\frac{1}{4|\xi|} \sin (2(\bar{t}-t)|\xi|)+\frac{1}{2|\xi|} \sin ((\bar{t}-t)|\xi|)-\frac{1}{4|\xi|} \sin (2 \bar{t}|\xi|) \\
-\frac{1}{4|\xi|} \sin (2 t|\xi|)-\frac{1}{2|\xi|} \sin ((\bar{t}+t)|\xi|)
\end{gathered}
$$

The change of variable $(\bar{t}-t) \xi=\eta$ yields

$$
\begin{aligned}
I_{1} & \geq \int_{|\xi| \geq(\bar{t}-t)^{-1}} d \xi\left|\xi_{1}\right|^{1-2 H_{1}}\left|\xi_{2}\right|^{1-2 H_{2}}\left|\xi_{3}\right|^{1-2 H_{3}} \frac{1}{|\xi|^{2}} \frac{1}{t} A(t, \bar{t}, \xi) \\
& \geq(\bar{t}-t)^{2 \bar{\kappa}} \int_{|\eta| \geq 1} d \eta\left|\eta_{1}\right|^{1-2 H_{1}}\left|\eta_{2}\right|^{1-2 H_{2}}\left|\eta_{3}\right|^{1-2 H_{3}} \frac{1}{|\eta|^{2}}(1-\cos |\eta|) \\
& \geq c_{1}|\bar{t}-t|^{2 \bar{\kappa}} .
\end{aligned}
$$

Similarly,

$$
\begin{aligned}
I_{2} & =\int_{|\xi| \geq(\bar{t}-t)^{-1}} d \xi\left|\xi_{1}\right|^{1-2 H_{1}}\left|\xi_{2}\right|^{1-2 H_{2}}\left|\xi_{3}\right|^{1-2 H_{3}} \frac{1}{|\xi|^{2}} B(t, \bar{t}, \xi) \\
& \geq-c_{1} \int_{|\xi| \geq(\bar{t}-t)^{-1}} d \xi\left|\xi_{1}\right|^{1-2 H_{1}}\left|\xi_{2}\right|^{1-2 H_{2}}\left|\xi_{3}\right|^{1-2 H_{3}} \frac{1}{|\xi|^{3}} \\
& \geq-c_{1}(\bar{t}-t)^{2 \bar{\kappa}+1} \int_{|\eta| \geq 1} d \eta \frac{1}{|\eta|^{3}}\left|\eta_{1}\right|^{1-2 H_{1}}\left|\eta_{2}\right|^{1-2 H_{2}}\left|\eta_{3}\right|^{1-2 H_{3}}
\end{aligned}
$$

Therefore,

$$
Z_{2}(t, \bar{t}, x) \geq c_{1} t|\bar{t}-t|^{2 \bar{\kappa}}-c_{2}^{\prime}|\bar{t}-t|^{2 \bar{\kappa}+1} \geq c_{1}^{\prime}|\bar{t}-t|^{2 \bar{\kappa}}
$$

when $|\bar{t}-t|$ is sufficiently small and $t \geq t_{0}$. So we conclude that

$$
\mathbb{E}\left(|u(t, x)-u(\bar{t}, x)|^{2}\right) \geq c_{1}|\bar{t}-t|^{2 \bar{\kappa}}
$$


On the other hand, we have

$$
\begin{aligned}
Z_{2}(t, \bar{t}, x) \leq c_{2} \int_{0}^{t} d s \int_{|\xi| \leq(\bar{t}-t)^{-1}} d \xi\left|\xi_{1}\right|^{1-2 H_{1}}\left|\xi_{2}\right|^{1-2 H_{2}}\left|\xi_{3}\right|^{1-2 H_{3}}(\bar{t}-t)^{2} \\
\quad+c_{2} \int_{0}^{t} d s \int_{|\xi| \geq(\bar{t}-t)^{-1}} d \xi\left|\xi_{1}\right|^{1-2 H_{1}}\left|\xi_{2}\right|^{1-2 H_{2}}\left|\xi_{3}\right|^{1-2 H_{3}} \frac{1}{|\xi|^{2}}
\end{aligned}
$$

Applying the substitution $\xi(\bar{t}-t)=\eta$ to both the above integrals, we see that

$$
Z_{2}(t, \bar{t}, x) \leq c_{2}|\bar{t}-t|^{2 \bar{\kappa}}
$$

Thus (b) is proved.

Combining the upper bound in (6.10) and (6.11), taking into account that the process $u$ is Gaussian and applying Kolmogorov continuity criterion, for any $\delta>0$ and any bounded rectangle $I \subset \mathbb{R}^{3}$, there is a random variable $c_{\delta, I}$ such that almost surely

$$
|u(s, x)-u(t, y)| \leq c_{\delta, I}\left(|s-t|^{\bar{\kappa}-\delta}+|x-y|^{\bar{\kappa}-\delta}\right)
$$

The first inequalities of (6.10) and (6.11) tell us that the exponent $\bar{\kappa}$ is the optimal.

Remark 6.3 Theorem 5.1 in [5] shows that the result obtained in Sect. 5.2 is optimal. The result in Theorem 6.2 suggests that the result in Theorem 6.1 may not be optimal. To prove the result is optimal or to find the optimal result needs further research.

\section{Appendix}

In this section we prove some lemmas used in this paper.

Lemma 6.4 For any $s \geq t$

$$
(G(s) * G(t))(d x)=\frac{1}{8 \pi|x|} \mathbf{1}_{[s-t, s+t]}(|x|) d x .
$$

Proof To calculate $(G(t) * G(s))(d x)$, let us consider two independent random variables $X$ and $Y$ uniformly distributed on the spheres with radii $s$ and $t$ respectively with $s \geq t$. Note that the distribution of $X+Y$ is rotationally invariant. Consider a bounded continuous function $\varphi$ on $\mathbb{R}$. We have

$$
\mathbb{E}(\varphi(|X+Y|))=\frac{1}{(4 \pi)^{2}} \int_{S^{2}} \int_{S^{2}} \varphi(|s x+t y|) \sigma(d y) \sigma(d x) .
$$


It is easy to see that in the above expression, the integral with respect to $\sigma(d y)$ does not depend on $x$, so we can take $x=x_{0}=(0,0,1)$, and using spherical coordinates $y=(\sin \phi \cos \theta, \sin \phi \sin \theta, \cos \phi)$, we have

$$
\begin{aligned}
\mathbb{E}(\varphi(|X+Y|)) & =\frac{1}{4 \pi} \int_{S^{2}} \varphi\left(\left|s x_{0}+t y\right|\right) \sigma(d y) \\
& =\frac{1}{2} \int_{0}^{\pi} \varphi\left(\sqrt{s^{2}+t^{2}+2 t s \cos \phi}\right) \sin \phi d \phi .
\end{aligned}
$$

Making the change of variable $u=\sqrt{s^{2}+t^{2}+2 t s \cos \phi}$, we obtain

$$
\mathbb{E}(\varphi(|X+Y|))=\frac{1}{2 t s} \int_{s-t}^{s+t} \varphi(u) u d u=\frac{1}{2 t s} \int_{B(0, s+t) \backslash B(0, s-t)} \varphi(|z|) \frac{1}{4 \pi|z|} d z,
$$

where $B(0, r)$ is the ball in $\mathbb{R}^{3}$ with center 0 and radius $r$. So we conclude that the random variable $X+Y$ has a density given by

$$
\rho(z)=\frac{1}{8 \pi t s|z|} \mathbf{1}_{[s-t, s+t]}(|z|) .
$$

Taking into account that the distributions of $X$ and $Y$ are given by $\frac{1}{s} G(s, d x)$ and $\frac{1}{t} G(t, d x)$ respectively, we easily get the desired result (6.15).

Our next result gives an integral identity which is used a lot in this paper. See also Theorem 5.2 in [5] for a similar result.

The following result is related to Theorem 5.2 in [5].

Lemma 6.5 Let $\varphi$ and $\psi$ be two bounded Borel measurable functions and assume that (2.5) holds and $s \geq t>0$. Then for any $w \in \mathbb{R}^{3}$ we have

$$
\begin{aligned}
& \int_{\mathbb{R}^{3}} \int_{\mathbb{R}^{3}} \varphi(x) G(t, d x) \psi(y) G(s, d y) f(x-y+w) \\
& =\int_{\mathbb{R}^{3}} \mathcal{F}(\varphi G(t))(\xi) \overline{\mathcal{F}(\psi G(s))(\xi)} e^{-i w \cdot \xi} \mu(d \xi) .
\end{aligned}
$$

Proof Let $\phi(x)=C \exp \left(\frac{1}{|x|^{2}-1}\right) \mathbf{1}_{[0,1)}(|x|)$, where $C$ is a normalization coefficient such that $\int_{\mathbb{R}^{3}} \phi(x) d x=1$. Set $\phi_{\varepsilon}(x)=\frac{1}{\varepsilon^{3}} \phi\left(\frac{x}{\varepsilon}\right)$, with $\varepsilon \leq t$. Using the Fourier transform we have 


$$
\begin{aligned}
\int_{\mathbb{R}^{3}} & \left((\varphi G(t) * \widehat{\psi G(s)}) * \phi_{\varepsilon}\right)(x) f(x+w) d x \\
\quad= & \int_{\mathbb{R}^{3}} \mathcal{F}(\varphi G(t))(\xi) \overline{\mathcal{F}(\psi G(s))(\xi)}(\mathcal{F} \phi)(\varepsilon \xi) e^{-i w \cdot \xi} \mu(d \xi),
\end{aligned}
$$

where $\widetilde{\varphi G(t)}(x)=\varphi(-x) G(t,-d x)$. We are going to show that

$$
\left|\left((\varphi G(t) * \widetilde{\psi G(s)}) * \phi_{\varepsilon}\right)(x)\right| \leq \frac{C}{|x|} \mathbf{1}_{[0,3 s]}(|x|) .
$$

Indeed, since $\varphi$ and $\psi$ are bounded, we have

$$
\begin{aligned}
\left|\left((\varphi G(t) * \widetilde{\psi G(s)}) * \phi_{\varepsilon}\right)(x)\right| & \leq C\left((G(t) * G(s)) * \phi_{\varepsilon}\right)(x) \\
& =C\left(\frac{1}{|\cdot|} \mathbf{1}_{[s-t, s+t]}(|\cdot|) * \phi_{\varepsilon}\right)(x) .
\end{aligned}
$$

Note first that the function

$$
\left(\left(\frac{1}{|\cdot|} \mathbf{1}_{[s-t, s+t]}(|\cdot|)\right) * \phi_{\varepsilon}\right)(x)
$$

is supported within a ball centered at the origin with radius $3 s$ for every $\varepsilon \leq t$ and it converges to $\frac{1}{|z|} \mathbf{1}_{[s-t, s+t]}$ almost everywhere. Next, for $|x| \leq 3 s$ we have

$$
\begin{aligned}
\left(\left(\frac{1}{|\cdot|} \mathbf{1}_{[s-t, s+t]}(|\cdot|)\right) * \phi_{\varepsilon}\right)(x) & =\int_{\mathbb{R}^{3}} \frac{1}{|x-z|} \mathbf{1}_{[s-t, s+t]}(|x-z|) \phi_{\varepsilon}(z) d z \\
& \leq \int_{|x-z| \geq \frac{|x|}{2}} \frac{1}{|x-z|} \phi_{\epsilon}(z) d z+\int_{|x-z|<\frac{|x|}{2}} \frac{1}{|x-z|} \phi_{\epsilon}(z) d z \\
& =\frac{2}{|x|}+\int_{|z|<\frac{|x|}{2}} \frac{1}{|z|} \phi_{\varepsilon}(z+x) d z,
\end{aligned}
$$

where in the second integral we have used the change of variable $z-x \rightarrow z$. Since in the second integral $|z|<\frac{|x|}{2}$, we have $|z+x| \geq|x|-|z| \geq \frac{|x|}{2}$, and

$$
\int_{|z|<\frac{|x|}{2}} \frac{1}{|z|} \phi_{\varepsilon}(z+x) \leq \int_{|z|<\frac{|x|}{2}} \frac{1}{|z|} \phi_{\varepsilon}\left(\frac{x}{2}\right) d z=C|x|^{2} \phi_{\varepsilon}\left(\frac{x}{2}\right) \leq C \frac{1}{|x|}
$$


where in the last inequality we used the fact that $\sup _{x \in \mathbb{R}^{3}}|x|^{3} \phi(x)<\infty$. So (6.19) is proved. Then by an application of the dominated convergence theorem we have

$$
\begin{aligned}
& \lim _{\varepsilon \rightarrow 0} \int_{\mathbb{R}^{3}}\left((\varphi G(t) * \widetilde{\psi G(s)}) * \phi_{\varepsilon}\right)(x) f(x+w) d x \\
= & \int_{\mathbb{R}^{3}}(\varphi G(t) * \widetilde{\psi G(s)})(x) f(x+w) d x \\
= & \int_{\mathbb{R}^{3}} \int_{\mathbb{R}^{3}} \varphi(x) G(t, d x) \varphi(y) G(s, d y) f(x-y+w) .
\end{aligned}
$$

On the other hand, the estimate (6.19) implies that the quantity in (6.18) is uniformly bounded in $\varepsilon$. Hence, by Fatou's lemma $\int_{\mathbb{R}^{3}}|\mathcal{F}(\varphi G(t))(\xi)||\mathcal{F}(\psi G(s))(\xi)| \mu(d \xi)<$ $\infty$, and by the dominated convergence, the right-hand side of (6.18) converges to

$$
\int_{\mathbb{R}^{3}} \mathcal{F}(\varphi G(t))(\xi) \overline{\mathcal{F}(\psi G(s))(\xi)} e^{-i w \cdot \xi} \mu(d \xi) .
$$

This completes the proof of the lemma.

In particular, if in the above lemma, take $\varphi=\psi, t=s$ and $w=0$, then for any $t>0$ we have

$$
\int_{\mathbb{R}^{3}} \int_{\mathbb{R}^{3}} \varphi(x) G(t, d x) \varphi(y) G(t, d y) f(x-y)=\int_{\mathbb{R}^{3}}|\mathcal{F}(\varphi G(t))(\xi)|^{2} \mu(d \xi) .
$$

More specifically, if in addition, we take $\varphi \equiv 1$, then we obtain

$$
\int_{\mathbb{R}^{3}} \int_{\mathbb{R}^{3}} G(t, d x) G(t, d y) f(x-y)=\int_{\mathbb{R}^{3}} \frac{(\sin (t|\xi|))^{2}}{|\xi|^{2}} \mu(d \xi) .
$$

Acknowledgments We would like to thank the anonymous referees for their many constructive and detailed comments which helped to improve this work. D. Nualart is supported by the NSF Grant DMS1208625. Y. Hu is partially supported by a Grant from the Simons Foundation \#209206.

\section{References}

1. Balan, R., Tudor, C.: The stochastic heat equation with fractional-colored noise: existence of the solution. ALEA Lat. Am. J. Probab. Math. Stat. 4, 57-87 (2008)

2. Balan, R., Tudor, C.: Stochastic heat equation with multiplicative fractional-colored noise. J. Theoret. Probab. 23(3), 834-870 (2010)

3. Dalang, R.C., Sanz-Solé, M.: Hölder-Sobolev Regularity of the Solution to the Stochastic Wave Equation in Dimension Three. Memoirs of the American Mathematical Society Number 931, May (2009)

4. Dalang, R.C., Quer-Sardanyons, L.: Stochastic integrals for spde's: a comparison. Expositiones Mathematicae 29(1), 67-109 (2011) 
5. Khoshnevisan, D., Xiao, Y.: Harmonic analysis of additive Lévy processes. Probab. Theory Related Fields 145(3-4), 459-515 (2009)

6. Nualart, D., Rovira, C., Tindel, S.: Probabilistic models for vortex filaments based on fractional Brownian motion. Ann. Probab. 31(4), 1862-1899 (2003)

7. Sogge, C.D.: Lectures on Nonlinear Wave Equations. Monographs in Analysis. International Press, Boston (1995)

8. Walsh, J.B.: An introduction to stochastic partial differential equations, École d'été de Probabilités de Saint Flour XIV, -1984, Lecture Notes in Mathematics, vol. 1180, pp. 265-439. Springer, Berlin (1986) 